The Astrophysical JouRnal, 523:654-677, 1999 October 1

(C) 1999. The American Astronomical Society. All rights reserved. Printed in U.S.A.

\title{
THE SPITE LITHIUM PLATEAU: ULTRATHIN BUT POSTPRIMORDIAL ${ }^{1}$
}

\author{
SEAN G. RYAN \\ Royal Greenwich Observatory (closed), and Physics Department, The Open University, Walton Hall, Milton Keynes MK7 6AA, England, UK; \\ s.g.ryan@open.ac.uk \\ JOHN E. NORRIS \\ Research School of Astronomy and Astrophysics, The Australian National University, Private Bag, Weston Creek Post Office, ACT 2611, Australia; \\ jen@mso.anu.edu.au \\ AND \\ Timothy C. BeERS \\ Department of Physics and Astronomy, Michigan State University, East Lansing, MI 48824; beers@pa.msu.edu \\ Received 1999 March 1; accepted 1999 May 13
}

\begin{abstract}
We have studied 23 very metal-poor field turnoff stars, specifically chosen to enable a precise measurement of the dispersion in the lithium abundance of the Spite Li plateau. We concentrated on stars having a narrow range of effective temperature and very low metallicities $([\mathrm{Fe} / \mathrm{H}] \lesssim-2.5)$ to reduce the effects of systematic errors and have made particular efforts to minimize random errors. A typical statistical error for our abundances is 0.033 dex $(1 \sigma)$, which represents a factor of 2 improvement on most previous studies. Our sample does not exhibit a trend with effective temperature, although the temperature range is limited. However, for $-3.6<[\mathrm{Fe} / \mathrm{H}]<-2.3$ we do recover a dependence on metallicity at $d A(\mathrm{Li}) / d[\mathrm{Fe} / \mathrm{H}]=0.118 \pm 0.023(1 \sigma)$ dex per dex, almost the same level as discussed previously. Earlier claims for a lack of dependence of $A(\mathrm{Li})$ on abundance are shown to have arisen probably from noisier estimates of effective temperatures and metallicities, which have erased the real trend. The dependence is concordant with theoretical predictions of Galactic chemical evolution (GCE) of Li (even in such metal-poor stars) and with the published level of ${ }^{6} \mathrm{Li}$ in two of the stars of our sample, which we use to infer the GCE ${ }^{7} \mathrm{Li}$ contribution. One of the 23 stars, G186-26, was known already to be strongly Li-depleted. Of the remaining 22 objects, 21 have abundances consistent with an observed spread about the metallicity trend of a mere $0.031 \mathrm{dex}(1 \sigma)$. Because the formal errors are $0.033 \mathrm{dex}$, we conclude that the intrinsic spread is effectively zero at the very metal-poor halo turnoff. This is established at much higher precision than previous studies $(\sim 0.06-0.08 \mathrm{dex})$. The essentially zero intrinsic spread leads to the conclusion that either these stars have all changed their surface Li abundances very uniformly, or else they exhibit close to the primordial abundance sought for its cosmological significance. We cannot rule out a uniform depletion mechanism, but economy of hypothesis supports the latter interpretation. The lack of spread in the $A(\mathrm{Li})$ abundances limits permissible depletion by rotationally induced mixing models to less than 0.1 dex. Correcting for the GCE contribution to both ${ }^{6} \mathrm{Li}$ and ${ }^{7} \mathrm{Li}$, we infer a primordial abundance $A(\mathrm{Li})_{p} \simeq 2.00 \mathrm{dex}$, with three systematic uncertainties of up to 0.1 dex each depending on uncertainties in the effective temperature scale, stellar atmosphere models, and correction for GCE. (This value rests on an effective-temperature zero-point set by Magain's and Bell \& Oke's $b-y$ calibrations of metal-poor stars and the model atmospheres without convective overshoot.) We predict that observations of $\mathrm{Li}$ in extremely low-metallicity stars, having $[\mathrm{Fe} / \mathrm{H}]<-3$, will yield smaller $A(\mathrm{Li})$ values than the bulk of stars in this sample, consistent with a low primordial abundance. The difference between our field star observations and published M92 data suggests real field-to-cluster differences. This may indicate different angular momentum evolutionary histories, with interactions between protostellar disks in the dense globular cluster environments possibly being responsible. Further study of $\mathrm{Li}$ in globular clusters and in very metal-poor field samples is required to clarify the situation.
\end{abstract}

Subject headings: Galaxy: halo - nuclear reactions, nucleosynthesis, abundances stars: abundances - stars: Population II

\section{INTRODUCTION}

Beginning with Spite \& Spite (1982), many authors have used the apparent uniformity of the abundance of lithium in the atmospheres of metal-poor $([\mathrm{Fe} / \mathrm{H}]<-1.0)$ subdwarfs warmer than $T_{\text {eff }}=5600 \mathrm{~K}$ to infer the primordial value generated by standard Big Bang nucleosynthesis. By

\footnotetext{
${ }^{1}$ Based on observations obtained with the University College London echelle spectrograph (UCLES) on the $3.9 \mathrm{~m}$ Anglo-Australian Telescope (AAT), the Double Beam Spectrograph (DBS) on the Australian National University $2.3 \mathrm{~m}$ telescope, and the Utrecht echelle spectrograph (UES) on the $4.2 \mathrm{~m}$ William Herschel Telescope (WHT).
}

restricting a sample to $T_{\text {eff }}>5600 \mathrm{~K}$, one avoids welldocumented processes that alter the surface $\mathrm{Li}$ abundance in cooler dwarf stars (e.g., Deliyannis, Demarque, \& Kawaler 1990). If this interpretation is correct, the so-called "Spite Li Plateau" abundance of $A(\mathrm{Li})=12+\lg [N(\mathrm{Li}) /$ $N(\mathrm{H})] \simeq 2.1$ provides constraints on the baryon-to-photon ratio in the early universe and hence $\Omega_{b}$ (e.g., Deliyannis 1995). ${ }^{2}$

However, several theoretical and observational results have cast doubt on use of the observed Li plateau abun-

${ }^{2} \lg X=\log _{10} X$ 
TABLE 1

Photometry for Program Stars

\begin{tabular}{|c|c|c|c|c|c|c|c|c|c|c|c|c|c|c|c|c|c|c|}
\hline \multirow[b]{2}{*}{$\begin{array}{c}\text { STAR } \\
\text { (1) }\end{array}$} & \multicolumn{2}{|c|}{$(1950)$} & \multirow[b]{2}{*}{$\begin{array}{l}V \\
\text { (4) }\end{array}$} & \multirow[b]{2}{*}{$\begin{array}{c}B-V \\
(5)\end{array}$} & \multirow[b]{2}{*}{$\begin{array}{c}V-R \\
(6)\end{array}$} & \multirow[b]{2}{*}{$\begin{array}{c}R-I_{\mathrm{C}} \\
\text { (7) }\end{array}$} & \multirow[b]{2}{*}{$\begin{array}{c}E(B V) \\
(8)\end{array}$} & \multirow[b]{2}{*}{$\begin{array}{l}n_{J} \\
(9)\end{array}$} & \multirow[b]{2}{*}{$\begin{array}{c}b-y \\
(10)\end{array}$} & \multirow[b]{2}{*}{$\begin{array}{c}c_{1} \\
(11)\end{array}$} & \multirow[b]{2}{*}{$\begin{array}{l}E(b y) \\
(12)\end{array}$} & \multirow[b]{2}{*}{$\begin{array}{c}n_{S} \\
(13)\end{array}$} & \multirow[b]{2}{*}{$\begin{array}{c}\beta \\
(14)\end{array}$} & \multirow[b]{2}{*}{$\begin{array}{c}n_{\beta} \\
(15)\end{array}$} & \multirow[b]{2}{*}{$\begin{array}{c}\text { REFERENCES } \\
(16)\end{array}$} & \multirow[b]{2}{*}{$\begin{array}{l}\text { HP2 } \\
(17)\end{array}$} & \multirow[b]{2}{*}{$\begin{array}{c}E(B-V) \\
\quad(18)\end{array}$} & \multirow[b]{2}{*}{$\begin{array}{l}\text { Notes } \\
(19)\end{array}$} \\
\hline & $\begin{array}{l}\text { R.A. } \\
\text { (2) }\end{array}$ & $\begin{array}{l}\text { Decl. } \\
\text { (3) }\end{array}$ & & & & & & & & & & & & & & & & \\
\hline LP $651-4 \ldots \ldots \ldots \ldots \ldots$ & 024142 & -053930 & 12.04 & 0.393 & 0.271 & 0.305 & 0.02 & 2 & 0.321 & 0.340 & 0.043 & 4 & 2.615 & 4 & 5,12 & 4.49 & 0.030 & $\ldots$ \\
\hline G4-37 (Tou 23:443) ........... & 024155 & +081618 & 11.42 & 0.47 & $\ldots$ & $\ldots$ & 0.04 & 2 & 0.363 & 0.306 & 0.063 & 6 & 2.614 & 7 & $1,6,7,8,12$ & 4.22 & 0.054 & $\ldots$ \\
\hline LP $831-70 \ldots \ldots \ldots \ldots \ldots \ldots$ & 030352 & -223048 & 11.62 & 0.400 & 0.273 & 0.309 & 0.00 & 2 & 0.326 & 0.262 & 0.014 & 4 & 2.602 & 4 & 5,12 & 4.05 & 0.00 & $\ldots$ \\
\hline $\mathrm{CD}-33^{\circ} 1173 \ldots \ldots \ldots \ldots \ldots$ & 031734 & -330124 & 10.94 & 0.364 & 0.255 & 0.285 & 0.00 & 2 & 0.292 & 0.356 & 0.011 & 5 & 2.618 & 5 & 5,12 & 4.91 & 0.00 & $\ldots$ \\
\hline $\mathrm{BD}+3^{\circ} 740(\mathrm{G} 84-29) \ldots \ldots \ldots$ & 045838 & +040224 & 9.80 & 0.36 & $\ldots$ & $\ldots$ & 0.01 & 3 & 0.315 & 0.365 & 0.028 & 5 & 2.616 & 5 & $1,6,7,8,12$ & 4.61 & 0.015 & $\ldots$ \\
\hline $\mathrm{BD}+24^{\circ} 1676(\mathrm{G} 88-32) \ldots \ldots$. & 072739 & +241142 & 10.80 & 0.36 & $\ldots$ & $\ldots$ & 0.01 & 3 & 0.311 & 0.356 & 0.00 & 3 & 2.591 & 3 & $1,6,7,8$ & 4.35 & 0.00 & $\ldots$ \\
\hline $\mathrm{BD}+20^{\circ} 2030(\mathrm{G} 40-14) \ldots \ldots$. & 081313 & +195124 & 11.20 & 0.38 & $\ldots$ & $\ldots$ & 0.01 & 3 & 0.312 & 0.322 & 0.021 & 6 & 2.618 & 6 & $1,6,10,12$ & 4.55 & 0.010 & SB1(Ref.1) \\
\hline $\mathrm{BD}+9^{\circ} 2190(\mathrm{G} 41-41) \ldots \ldots \ldots$ & 092635 & +085124 & 11.15 & 0.38 & $\ldots$ & $\ldots$ & 0.02 & 4 & 0.307 & 0.379 & 0.023 & 5 & 2.615 & 5 & $1,6,7,8,12$ & 4.83 & 0.016 & $\ldots$ \\
\hline $\mathrm{BD}+1^{\circ} 2341 \mathrm{p}(\mathrm{G} 48-29) \ldots \ldots$. & 093808 & +011436 & 10.47 & 0.38 & $\ldots$ & 0.29 & 0.02 & 5 & 0.298 & 0.351 & 0.014 & 9 & 2.620 & 6 & $1,3,6,7,8,12$ & 4.92 & 0.010 & LHS 2169, LP $608-62$ \\
\hline HD $84937\left(B D+14^{\circ} 2151\right) \ldots$ & 094617 & +135918 & 8.33 & 0.39 & $\ldots$ & 0.31 & 0.01 & 5 & 0.303 & 0.354 & 0.013 & 9 & 2.613 & 8 & $1,2,6,7,8$ & 4.46 & 0.004 & G43-3, LHS 2194 \\
\hline BD $-13^{\circ} 3442 \ldots \ldots \ldots \ldots \ldots$ & 114418 & -134954 & 10.26 & 0.399 & 0.275 & 0.294 & 0.01 & 2 & 0.308 & 0.385 & 0.035 & 5 & 2.622 & 5 & 5,12 & 4.52 & 0.020 & $\ldots$ \\
\hline G64-12 (W1492) .............. & 133730 & +001254 & 11.47 & 0.38 & $\ldots$ & $\ldots$ & 0.00 & 6 & 0.307 & 0.337 & 0.023 & 7 & 2.617 & 8 & $1,6,7,8,12$ & 4.77 & 0.006 & $\ldots$ \\
\hline G64-37 (R841) ............... & 135953 & -052418 & 11.13 & 0.368 & 0.268 & 0.298 & 0.02 & 2 & 0.300 & 0.333 & 0.015 & 10 & 2.623 & 10 & $1,5,6,7,8,12$ & 4.75 & 0.011 & $\ldots$ \\
\hline $\mathrm{BD}+26^{\circ} 2621(\mathrm{G} 166-54) \ldots \ldots$ & 145200 & +254612 & 11.05 & 0.41 & $\ldots$ & & 0.00 & 1 & 0.324 & 0.322 & 0.034 & 11 & 2.619 & 13 & 1,12 & 4.66 & 0.014 & $\ldots$ \\
\hline $\mathrm{CD}-71^{\circ} 1234 \ldots \ldots \ldots \ldots \ldots$ & 160218 & -711400 & 10.44 & 0.412 & 0.277 & 0.321 & 0.04 & 2 & 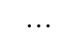 & $\ldots$ & $\ldots$ & $\ldots$ & $\ldots$ & $\ldots$ & 5 & 4.23 & 0.040 & SB1(Table 4) \\
\hline $\mathrm{BD}+26: 3578($ HD 338529) ... & 193029 & +261706 & 9.35 & 0.40 & $\ldots$ & $\ldots$ & 0.02 & 2 & 0.308 & 0.366 & 0.010 & 3 & 2.600 & 3 & $1,7,8$ & 4.48 & 0.007 & $\ldots$ \\
\hline 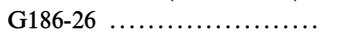 & 202237 & +245330 & 10.82 & 0.40 & $\ldots$ & $\ldots$ & 0.02 & 2 & 0.306 & 0.339 & 0.015 & 3 & 2.608 & 3 & $1,7,8$ & 4.59 & 0.010 & Ultra-Li-weak \\
\hline LP $635-14 \ldots \ldots \ldots \ldots \ldots$ & 202413 & -004700 & 11.33 & 0.426 & 0.279 & 0.314 & 0.05 & 2 & 0.347 & 0.366 & 0.064 & 4 & 2.611 & 4 & 5,12 & 4.64 & 0.060 & $\ldots$ \\
\hline LP $815-43 \ldots \ldots \ldots \ldots \ldots \ldots$ & 203521 & -203630 & 10.91 & 0.384 & 0.262 & 0.296 & 0.04: & 2 & 0.304 & 0.382 & 0.033 & 4 & 2.623 & 4 & 5,12 & 4.95 & 0.033 & $\ldots$ \\
\hline CS $22943-095 \ldots \ldots \ldots \ldots \ldots$ & 203559 & -470052 & 11.76 & $0.39:$ & $\ldots$ & $\ldots$ & 0.00 & $\ldots$ & 0.324 & 0.335 & 0.036 & 3 & 2.619 & 3 & 4,9 & 4.31 & 0.015 & $\ldots$ \\
\hline CD $-35^{\circ} 14849$ (W13543) ..... & 213048 & -353912 & 10.57 & 0.405 & 0.279 & 0.306 & 0.01 & 2 & 0.321 & 0.293 & 0.014 & 4 & 2.603 & 2 & $5,7,8,11$ & 4.09 & 0.005 & $\ldots$ \\
\hline 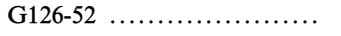 & 220151 & +191836 & 11.01 & 0.38 & $\ldots$ & $\ldots$ & 0.02 & 3 & 0.322 & 0.347 & 0.028 & 3 & 2.608 & 3 & $1,6,10$ & 4.62 & 0.020 & $\ldots$ \\
\hline $\mathrm{CD}-24^{\circ} 17504(\mathrm{G} 275-4) \ldots \ldots$ & 230439 & -240842 & 12.12 & 0.393 & 0.280 & 0.306 & 0.00 & 2 & 0.322 & 0.283 & 0.015 & 5 & 2.609 & 5 & 5,12 & 4.27 & .001 & $\cdots$ \\
\hline \multicolumn{19}{|c|}{ Standard Stars } \\
\hline HD $74000 \ldots \ldots \ldots \ldots \ldots \ldots$ & 083831 & -160936 & 9.64 & 0.42 & $\ldots$ & 0.31 & 0.00 & $\ldots$ & 0.311 & 0.295 & -0.01 & 7 & 2.596 & 4 & $1,3,7,8,11$ & 4.02 & 0.00 & $\ldots$ \\
\hline HD $140283 \ldots \ldots \ldots \ldots \ldots \ldots$ & 154022 & -104618 & 7.22 & 0.49 & $\ldots$ & $\ldots$ & $\ldots$ & $\ldots$ & $\ldots$ & $\ldots$ & $\ldots$ & $\ldots$ & $\ldots$ & $\ldots$ & $\ldots$ & 2.76 & $\ldots$ & $\ldots$ \\
\hline HD $160617 \ldots \ldots \ldots \ldots \ldots$ & $\ldots$ & $\ldots$ & $\ldots$ & $\ldots$ & $\ldots$ & $\ldots$ & $\ldots$ & $\ldots$ & $\ldots$ & $\ldots$ & $\ldots$ & $\ldots$ & $\ldots$ & $\ldots$ & $\ldots$ & 3.28 & $\ldots$ & Super-N-rich \\
\hline
\end{tabular}

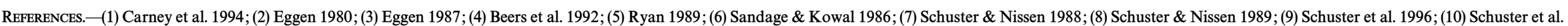
1993; (11) Sandage \& Fouts 1987; (12) W. J. Schuster 1998, private communication. 
dance as the primordial value. Lithium is fragile, and some stellar evolutionary models show that Li could have been depleted by an order of magnitude from a high primordial value and still attained plateau-like abundances by the age of the halo (e.g., Pinsonneault, Deliyannis, \& Demarque 1992). More recent computations by Pinsonneault et al. (1998), using an improved treatment of angular momentum evolution and comparisons with more modern observations, have reduced the permissible ${ }^{7} \mathrm{Li}$ depletion to the range $0.2-0.4$ dex. Trends of lithium abundance with $T_{\text {eff }}$ and $[\mathrm{Fe} / \mathrm{H}]$ (effectively a tilted plateau) have also been measured (Thorburn 1994; Norris, Ryan, \& Stringfellow 1994; Ryan et al. 1996a), which would not exist if the $\mathrm{Li}$ were primordial, although these results may be driven by larger than expected systematic errors in the effective temperatures (Bonifacio \& Molaro 1997). The huge ( $>1$ dex) Li deficiencies in some stars that are otherwise indistinguishable from normal plateau stars (Hobbs, Welty, \& Thorburn 1991; Thorburn 1994; Norris et al. 1997a; Ryan, Norris, \& Beers 1998) highlight the incompleteness of our understanding of Li processing in halo stars.

A direct challenge to the thesis of a primordial and therefore uniform Li plateau was mounted by Deliyannis, Pinsonneault, \& Duncan (1993) and supported by Thorburn (1994), who argued that the spread in measured plateau-star abundances exceeds that expected from observational errors. Deliyannis et al. tabulated a range of dispersions, depending on the characteristics of the sample, but with a minimum spread of $\sigma=0.04 \mathrm{dex}$, while Thorburn (1994) found a value around 0.1 dex for a much larger sample. Both groups concluded that Li production and/or depletion mechanisms had operated prior to the birth or during the evolution of the stars, in which case the measured Li abundance would not reflect solely that from Big Bang nucleosynthesis. Large ranges in $\mathrm{Li}$ abundance have also been deduced for subgiants in M92 (Deliyannis, Boesgaard, \& King 1995; Boesgaard et al. 1998). As further evidence of star-to-star differences in the halo field, Ryan et al. (1996a) cited the three stars G64-12, G64-37, and CD - 33 ${ }^{\circ} 1173$, all of which have extremely low metallicities $([\mathrm{Fe} / \mathrm{H}]<-3)$, are apparent nonbinaries, have surface temperatures $T_{\text {eff }} \approx$ $6250 \mathrm{~K}$, but for which they computed abundances $A(\mathrm{Li})=2.29 \pm 0.05,2.01 \pm 0.04$, and $1.89 \pm 0.06$, respectively.

However, the case for a measurable dispersion in the $\mathrm{Li}$ plateau has not gone unchallenged. Most recently, Molaro, Primas, \& Bonifacio (1995), Spite et al. (1996), and Bonifacio \& Molaro (1997) have questioned whether some of the error estimates in earlier works were realistic and have suggested that the dispersion is no greater than 0.08-0.10 dex, less than that found by Thorburn (1994) but not excluding the smaller scatter of Deliyannis et al. (1993). Ryan et al. (1996a) noted that most, but not all, published measurements could be reconciled within their claimed errors, thus illustrating that some error estimates were optimistic, a result that biases one toward overinterpreting the spread about the mean plateau value.

We set out to provide a substantially more accurate assessment of scatter about the Li plateau, to see whether we could rule out a purely primordial interpretation or whether the width was essentially consistent with small uncertainties in the measurements and analysis. We note at the outset that a very thin plateau is a necessary but not sufficient condition for the observed abundance to be pri- mordial. The Li plateau may be of infinitesimal width but depend on effective temperature and/or metallicity, in which case it will still not provide the primordial abundance, although some stars may be very close to it.

\section{DEFINITION OF THE SAMPLE}

We sought very high signal-to-noise ratio $(\mathrm{S} / \mathrm{N})$ measurements of the $\mathrm{Li} \lambda 6707$ doublet in a group of well-selected halo stars, with the aim of measuring $\mathrm{Li}$ abundances to higher precision than had been routinely accomplished previously. Estimates of the effective temperatures of stars are notoriously uncertain, particularly absolute as opposed to relative estimates, yet derived $\mathrm{Li}$ abundances depend on temperature. For example, the existence of temperaturedependent trends in the Li plateau depends on which effective temperature scale is adopted (compare Ryan et al. 1996a and Bonifacio \& Molaro 1997). Uncertainties in starto-star abundance comparisons also increase if their temperatures differ, because stellar atmosphere structures and color-effective temperature transformations also depend on temperature. To minimize the effects of systematic errors, we restricted our sample to a very narrow range in $T_{\text {eff }}$ and chose a narrow metallicity regime $([\mathrm{Fe} / \mathrm{H}] \lesssim-2.5)$, since this avoids possible metallicity-dependent errors in the color-effective-temperature transformation and model stellar atmospheres. Our sample targeted effective temperatures in the range $6100 \pm 50 \mathrm{~K}<T_{\text {eff }}<6300 \pm 50 \mathrm{~K}$ and metallicity in the range $-3.5 \leq[\mathrm{Fe} / \mathrm{H}] \leq-2.5$. We also restricted our sample to stars brighter than $V=13$ because of the requirement for high $\mathrm{S} / \mathrm{N}$.

The narrow temperature range places the stars at the turnoff of an old main-sequence population, effectively eliminating subgiants that spent their main-sequence lives at higher temperatures than those now observed and hence have different evolutionary histories. Another benefit of this restriction is that the surface gravity of the sample covers only a narrow range at the turnoff, although it has been noted many times previously that the $\mathrm{Li}$ abundance derived for halo dwarfs is quite insensitive to surface gravity errors. The use of very low-metallicity stars means that we are sampling material that has undergone a minimum of nucleosynthetic processing since the Big Bang.

We developed a target list of approximately 30 stars from the surveys of Schuster \& Nissen (1988), Ryan (1989), Beers, Preston, \& Shectman (1992), and Carney et al. (1994). A large sample was sought to reduce the impact of one or two "pathological" objects, such as marginally depleted examples of the ultra-Li-depleted stars or unrecognized binaries. We hoped to make multiple measurements of each one to verify the repeatability and to provide a check for radial velocity variability.

With the available telescope time, 22 of the stars were observed, and these are presented in Table 1. Also included in the table is G186-26, a known ultra-Li-depleted star (Hobbs et al. 1991), which satisfied our selection criteria but which we chose not to reobserve since its surface Li deficiency is already well established. Its relevance to our work is as a reminder that at least some otherwise similar stars have depleted the vast majority of their $\mathrm{Li}$.

\section{BASIC DATA}

The stars have Johnson-Cousins photometry from a small number of sources referenced in Table 1, whose con- 
sistency and accuracy have already been established at $\sigma=0.010 \mathrm{mag}$ per observation for $B-V$ and $R-I_{\mathrm{C}}$, and $\sigma=0.007 \mathrm{mag}$ in $V-R_{\mathrm{C}}$ (Ryan 1989) ${ }^{3}$. Strömgren photometry from Schuster, Nissen, and collaborators (see references in table) is available for all but one star. Columns (9), (13), and (15) in the table give the number of $B V R I, u v b y$, and $\beta$ measurements, respectively. Multiple measurements improve the photometric accuracy, which is important in deriving effective temperatures for the stars. We reduce the Johnson errors to 0.007 (for $B-V$ and $R-I$ ) and 0.005 (for $V-R)$ for two or more observations. Where rounding errors of up to $0.005 \mathrm{mag}$ affect Johnson-Cousins colors quoted to only $0.01 \mathrm{mag}$, we adopt larger uncertainties0.015 (for $B-V$ and $R-I$ ) and 0.010 (for $V-R$ ) for single measurements, and 0.010 (for $B-V$ and $R-I$ ) and 0.007 (for $V-R$ ) for two or more measurements. Schuster \& Nissen (1988) quote mean errors less than 0.008 mag for $b-y$ and $0.011 \mathrm{mag}$ for $\beta$ where there are three observations per star. Given that all of our program stars have three or more Strömgren observations, we adopt these error estimates for our entire sample.

Estimates of the interstellar reddening have been obtained from two techniques. Values estimated from reddening maps (Lucke 1978; Burstein \& Heiles 1982) and Johnson photometric distances have been made by Carney et al. (1994) and Ryan (1989), and are listed in Table 1, column (8), as $E(B V)$. Strömgren photometry estimates of $E(b-y)$ are based on a comparison of the $b-y$ color and $\beta$ reddening-free index (Schuster \& Nissen 1989, their eq. [1]), and these values are tabulated under $E(b y)$ in column (12). Based on the central wavelengths of the bandpasses and a $1 / \lambda$ reddening law, a relation $E(b-y)=0.7 E(B-V)$ is expected. However, comparison of the $E(B-V)$ inferred from the Strömgren values with the map-based values shows that the former are higher by $0.020 \mathrm{mag}$. The Strömgren technique suggests a mean reddening for the sample of $\langle E(B-V)\rangle=0.035 \mathrm{mag}$, whereas the map-based values suggest $\langle E(B-V)\rangle=0.015$ mag. We lack solid evidence as to which reddening scale, map or Strömgren, is better, but because the sample is fairly bright, we expect the intrinsic reddening to be low, so reduce all Strömgren values of $E(B-V)$ by $0.020 \mathrm{mag}$ prior to averaging. ${ }^{4}$ Once this offset is taken into account, the rms error inferred for a single $E(B-V)$ estimate is $0.009 \mathrm{mag}$. This error is assumed to affect all dereddening vectors of nonzero magnitude. We adopt $E(V-R)=0.78 E(B-V)$ and $E(R-I)=0.82 E$ $(B-V)$, following Savage \& Mathis (1979).

Table 1 also records measurements of the $\mathrm{H} \delta$ line spectroscopic index, HP2, from Beers et al. (1999) supplemented with new, $1 \AA$ resolution, high $\mathrm{S} / \mathrm{N}$ determinations based on observations with the $2.3 \mathrm{~m}$ telescope on Siding Spring Mountain in 1998 March and September. This pseudoequivalent width index complements the $\beta$ index and helps establish the effective temperature scale (below). It has the benefit of being independent of reddening and essentially independent of metallicity for our metal-poor sample and of having better temperature sensitivity than $\beta$ for the temperatures of our sample.

\footnotetext{
${ }^{3}$ The subscript " $\mathrm{C}$ " indicates photometry on the Cousins system.

${ }^{4}$ We cannot discount the possibility that the Galaxy does indeed have a high local reddening. This has been suggested already by Schuster et al. (1996), who find, on the basis of Strömgren photometry, an average reddening of 0.036 within $30^{\circ}$ of the South Galactic Pole.
}

\section{SPECTROSCOPIC OBSERVATIONS AND DATA REDUCTION}

\subsection{Observational Program}

Previous investigations of the Li plateau (see $\S 1$ ) claimed the significance of spreads at levels $\sigma \sim 0.08-0.10 \mathrm{dex}$, but Deliyannis et al. (1993) showed that the Li spread could be as small as $\sigma=0.04$ dex, depending on which subsample of stars they analyzed. These values indicated that we would require accuracies of order $\leq 10 \%$, or $\leq 0.04$ dex, to clarify the situation. The equivalent width for the Li line in halo turnoff stars is $\sim 20 \mathrm{~m} \AA$, thus requiring $\sigma_{W} \sim<2 \mathrm{~m} \AA$. This in turn demanded high-resolution spectra $(R \sim 40,000$, which just resolves the $6707 \AA$ Li doublet) and high S/N.

Observations were made using the University College London echelle spectrograph (UCLES) at the coude focus of the Anglo-Australian Telescope (AAT), spread over four epochs (in many instances utilizing partial nights). Two different observers acquired the data as follows: 1996 August 28 (S. G. R.); 1997 August 18-23 (J. E. N.); 1998 April 8-10 (J. E. N.); and 1998 August 10-15 (S. G. R.). Although crossdispersed echelle spectra often have only limited spatial coverage, which makes the sky and scattered light level difficult to measure, we used UCLES with its 79 lines $\mathrm{mm}^{-1}$ grating, which gives a $14^{\prime \prime}$ slit length, thus providing an unambiguous background subtraction. The spectra are shown coadded for multiple epochs in Figure 1. For the northern star BD $+9^{\circ} 2190$, we had only one low $\mathrm{S} / \mathrm{N}(=85)$ measurement with the AAT, so we obtained a supplementary observation in service time with the Utrecht echelle spectrograph (UES) at the Nasmyth focus of the William Herschel Telescope, on 1998 November 5. The UES is almost identical to the UCLES.

The Li measurements (discussed in detail in $\S 4.2$ ) are presented in Table 2, where we tabulate for each epoch the $\mathrm{S} / \mathrm{N}$, the equivalent width, and the equivalent width error. The $\mathrm{S} / \mathrm{N}$ is taken as the lesser of that expected from Poisson photon statistics and the scatter actually measured about the continuum fit. The variance ${ }^{-1}$-weighted sum, $\bar{W}$, and error $\sigma_{\bar{W}}$ are also provided, as are two $[\mathrm{Fe} / \mathrm{H}]$ values and our estimates of the effective temperature (see $\S 5$ ). The first column of $[\mathrm{Fe} / \mathrm{H}]$ values is from the literature and derives from high- and/or medium-resolution spectroscopic observations, for which the errors are believed to be $\sigma \simeq 0.15 \mathrm{dex}$ (see references in table). The second $[\mathrm{Fe} / \mathrm{H}]$ entries were obtained by applying the calibration of Beers et al. (1999) to the $1 \AA$ resolution spectra from which the HP2 index was measured. This metallicity estimate is based on two factors: the strength of the strong Ca II K line at $3933 \AA$ and absorption by many weaker lines in the interval 4000-4285 $\AA$ as measured by the autocorrelation function. These measured quantities have been calibrated for stars of known $[\mathrm{Fe} / \mathrm{H}]$ using joint theoretical and empirical techniques, and for dwarfs with $[\mathrm{Fe} / \mathrm{H}]<-2.5$, the external error is $\sigma=0.25$ dex (Beers et al. 1999, their Table 7). The agreement between the two $[\mathrm{Fe} / \mathrm{H}]$ values is very good; we shall return to this point later in the discussion.

To obtain the precision needed to examine potentially small levels of scatter about the Spite Li plateau, it was clear that we would need very accurate equivalent width measurements. In an earlier work (Norris et al. 1994) we estimated our Li equivalent width uncertainties due to random noise as $\sigma(W)=150 /\left(\mathrm{S} / \mathrm{N}_{50}\right) \mathrm{m} \AA$, where the $\mathrm{S} / \mathrm{N}$ was per 50 $\mathrm{m} \AA$ pixel. In the current study we sum over a wider band to 


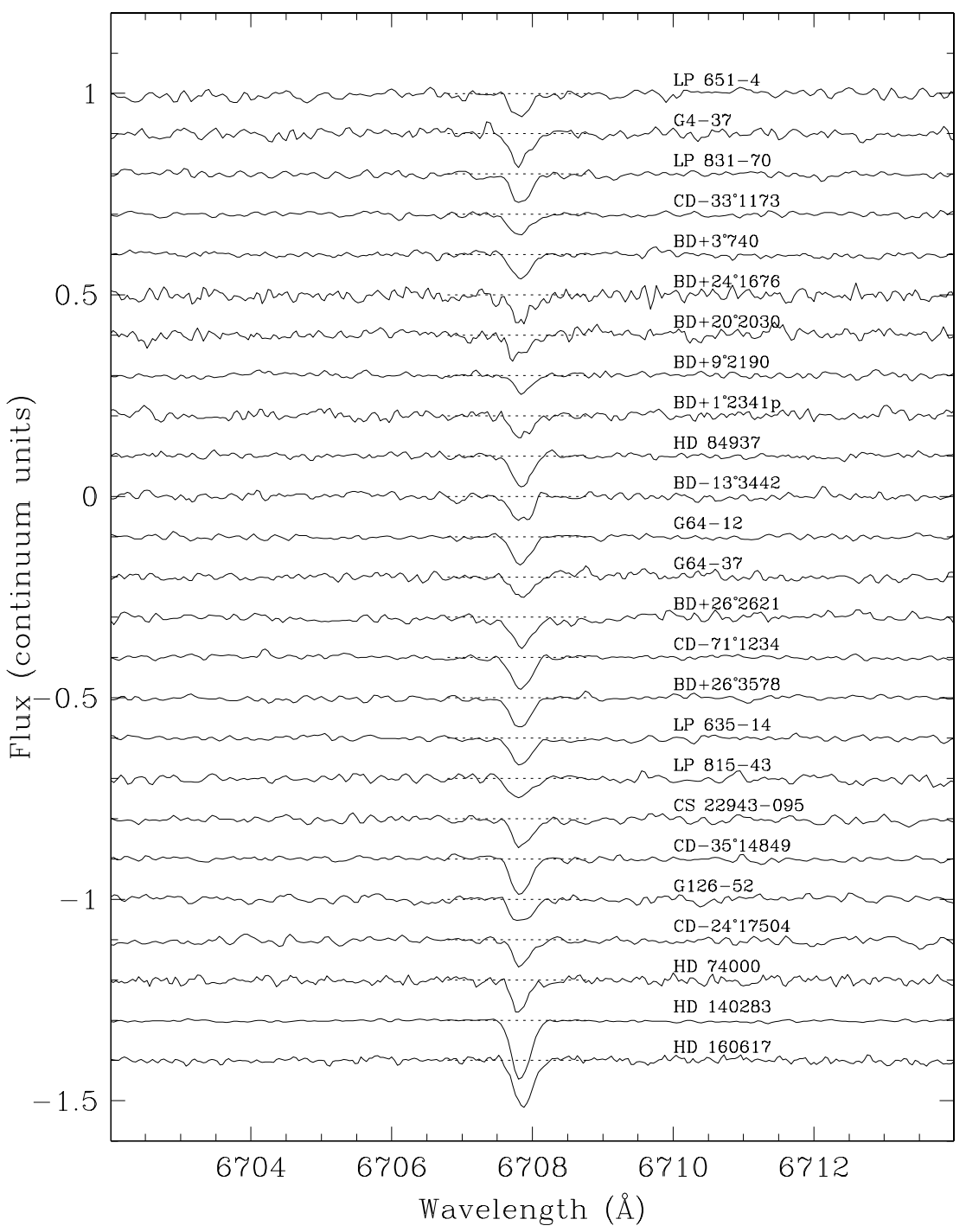

FIG. 1.-Spectra in region of the Li $6707 \AA$ Aine, offset by multiples of 0.1 continuum units. Multiple epochs have been coadded for this illustration, and the continuum location has been indicated with a dotted line, but actual measurements of equivalent widths were made for each epoch separately, to check repeatability. See text for details.

be more certain of including all of the line (see below) and so derive a larger numerator giving $\sigma(W)=184 /\left(\mathrm{S}_{\mathrm{N}} \mathrm{N}_{50}\right) \mathrm{m} \AA$. Because most epochs (except the single-night pilot run in 1996) have a $\mathrm{S} / \mathrm{N}$ in excess of 100 , we expected to achieve accuracies better than $2.0 \mathrm{~m} \AA$ per observation.

High absolute accuracy is harder to achieve than high internal precision. We discuss internal and external errors below, emphasizing that our primary requirement in studying scatter about the Li plateau is a large, homogeneously selected, consistently reduced, precisely measured, and consistently analyzed set of data. Several procedures were adopted to identify and minimize errors in order to meet this requirement. First we sought two epochs of data on each star to permit us to verify the repeatability of each measurement; we obtained multiple observations for 12 of the 22 stars. Second, all raw data were reduced by two of us independently, using different software. This allowed us to verify that the reduced spectra were consistent irrespective of which software, algorithms, and personal techniques were applied. Finally, two different techniques were used to measure the equivalent widths from the reduced spectra.

\subsection{Details on Equivalent Width Measurements}

\subsubsection{Continuum Placement}

The spectra of very metal-poor, main-sequence-turnoff stars are essentially devoid of lines over the range 6700$6715 \AA$ apart from the $6707 \AA \mathrm{Li}$ doublet itself. Even the $\mathrm{Ca}$ I line at $6717 \AA$ is invisible at the low metallicity and warm temperatures of many of these objects. (An equivalent width $W_{6717}<1 \mathrm{~mA}$ is expected for a model with $T_{\text {eff }}=$ $6000 \mathrm{~K}, \lg g=4.0$, and $[\mathrm{Fe} / \mathrm{H}]=-3$.) The continuum can therefore be defined accurately and objectively by fitting the mean flux on either side of the Li doublet. Two techniques for measuring equivalent widths are described in the following subsection. For the direct summation method, the continuum was computed as a quadratic fit to the flux in zones $2.5 \AA$ wide on either side of a $1.2 \AA$ wide zone of avoidance centered on the Li feature. The Gaussian-fitting technique determined its own continuum, using a linear interpolation between the flux in bands on either side of the Li feature, although the exact width of each continuum zone (approximately $4 \AA$ ) varied from star to star. 
TABLE 2

Equivalent Width and Abundance Data

\begin{tabular}{|c|c|c|c|c|c|c|c|c|c|c|c|c|c|c|c|c|c|c|c|c|c|}
\hline \multirow[b]{2}{*}{ STAR } & \multirow{2}{*}{$\begin{array}{c}{[\mathrm{Fe} / \mathrm{H}]} \\
\text { (literature) }\end{array}$} & \multirow[b]{2}{*}{ REFERENCES } & \multirow{2}{*}{$\begin{array}{c}{[\mathrm{Fe} / \mathrm{H}]} \\
(1 \AA)\end{array}$} & \multirow{2}{*}{$\begin{array}{l}T_{\text {eff }} \\
(\mathrm{K})\end{array}$} & \multirow{2}{*}{$\begin{array}{c}\sigma_{T} \\
(\mathrm{~K})\end{array}$} & \multicolumn{4}{|c|}{$\mathrm{S} / \mathrm{N}$ per $0.05 \AA ̊$ pixel } & \multicolumn{4}{|c|}{$\mathrm{W} \mathrm{m} \AA$} & \multicolumn{4}{|c|}{$\sigma_{W} \mathrm{~m} \AA$} & \multirow{2}{*}{$\begin{array}{c}\bar{W} \\
(\mathrm{~m} \AA)\end{array}$} & \multirow{2}{*}{$\begin{array}{c}\sigma_{\overline{\bar{W}}} \\
(\mathrm{~m} \AA)\end{array}$} & \multirow[b]{2}{*}{$A(\mathrm{Li})$} & \multirow[b]{2}{*}{$\sigma_{A(\mathrm{Li})}$} \\
\hline & & & & & & 96 & 97 & $98 \mathrm{~A}$ & $98 \mathrm{~B}$ & 96 & 97 & $98 \mathrm{~A}$ & 98B & 96 & 97 & $98 \mathrm{~A}$ & 98B & & & & \\
\hline LP $651-4$ & -2.96 & 1 & -2.60 & 6240 & 30 & 60 & 90 & $\ldots$ & 65 & 26.0 & 16.9 & $\ldots$ & 19.3 & 3.4 & 2.3 & $\ldots$ & 3.2 & 19.6 & 1.6 & 2.11 & 0.04 \\
\hline G4-37 & -2.73 & 1,2 & -2.70 & 6050 & 40 & $\ldots$ & 90 & $\ldots$ & $\ldots$ & $\ldots$ & 25.9 & $\ldots$ & $\ldots$ & $\ldots$ & 2.3 & $\ldots$ & $\ldots$ & 25.9 & 2.3 & 2.11 & 0.04 \\
\hline LP $831-70$ & -3.25 & 3 & -3.32 & 6050 & 20 & $\ldots$ & 105 & $\ldots$ & 100 & $\ldots$ & 20.7 & $\ldots$ & 25.7 & $\ldots$ & 2.0 & $\ldots$ & 2.1 & 23.1 & 1.4 & 2.07 & 0.03 \\
\hline $\mathrm{CD}-33^{\circ} 1173$ & -3.14 & 3 & -2.91 & 6250 & 20 & $\ldots$ & 115 & $\ldots$ & 130 & $\ldots$ & 18.5 & $\ldots$ & 16.2 & $\ldots$ & 1.8 & $\ldots$ & 1.6 & 17.2 & 1.2 & 2.06 & 0.03 \\
\hline $\mathrm{BD}+3^{\circ} 740$ & -2.78 & 2 & -2.70 & 6240 & 40 & $\ldots$ & $\ldots$ & 130 & 130 & $\ldots$ & $\ldots$ & 20.0 & 18.9 & $\ldots$ & $\ldots$ & 1.6 & 1.6 & 19.5 & 1.1 & 2.11 & 0.03 \\
\hline $\mathrm{BD}+24^{\circ} 1676$ & -2.71 & 2 & -2.38 & 6170 & 30 & $\ldots$ & $\ldots$ & 95 & $\ldots$ & $\ldots$ & $\ldots$ & 21.1 & $\ldots$ & $\ldots$ & $\ldots$ & 2.2 & $\ldots$ & 21.1 & 2.2 & 2.10 & 0.04 \\
\hline $\mathrm{BD}+20^{\circ} 2030$ & -2.71 & 2 & -2.64 & 6200 & 40 & $\ldots$ & $\ldots$ & 105 & $\ldots$ & $\ldots$ & $\ldots$ & 21.4 & $\ldots$ & $\ldots$ & $\ldots$ & 2.0 & $\ldots$ & 20.5 & 2.0 & 2.11 & 0.04 \\
\hline $\mathrm{BD}+9^{\circ} 2190$ & -2.89 & 1,2 & -2.83 & 6250 & 30 & $\ldots$ & $\ldots$ & 85 & $115^{\mathrm{a}}$ & $\ldots$ & $\ldots$ & 12.2 & $15.9^{\mathrm{a}}$ & $\ldots$ & $\ldots$ & 2.4 & $1.6^{\mathrm{a}}$ & 14.6 & 1.3 & 2.00 & 0.04 \\
\hline $\mathrm{BD}+1^{\circ} 2341 \mathrm{p}$ & -2.82 & 1,2 & -2.79 & 6260 & 40 & $\ldots$ & $\ldots$ & 130 & $\ldots$ & $\ldots$ & $\ldots$ & 17.8 & $\ldots$ & $\ldots$ & $\ldots$ & 1.6 & $\ldots$ & 17.8 & 1.6 & 2.09 & 0.04 \\
\hline HD 84937 & -2.30 & 1,2 & -2.12 & 6160 & 30 & $\ldots$ & $\ldots$ & 195 & $\ldots$ & $\ldots$ & $\ldots$ & 24.9 & $\ldots$ & $\ldots$ & $\ldots$ & 1.1 & $\ldots$ & 24.9 & 1.1 & 2.17 & 0.02 \\
\hline $\mathrm{BD}-13^{\circ} 3442$ & -2.99 & 3 & -2.79 & 6210 & 30 & $\ldots$ & $\ldots$ & 110 & 100 & $\ldots$ & $\ldots$ & 21.5 & 20.4 & $\ldots$ & $\ldots$ & 1.9 & 2.1 & 21.0 & 1.4 & 2.12 & 0.03 \\
\hline G64-12 & -3.17 & 3 & -3.24 & 6220 & 30 & $\ldots$ & 150 & $\ldots$ & 115 & $\ldots$ & 22.4 & $\ldots$ & 19.2 & $\ldots$ & 1.4 & $\ldots$ & 1.8 & 21.2 & 1.1 & 2.14 & 0.03 \\
\hline G64-37 & -3.23 & 3 & -3.15 & 6240 & 30 & $\ldots$ & 90 & 100 & $\ldots$ & $\ldots$ & 19.5 & 17.2 & $\ldots$ & $\ldots$ & 2.3 & 2.1 & $\ldots$ & 18.2 & 1.5 & 2.09 & 0.04 \\
\hline $\mathrm{BD}+26^{\circ} 2621$ & -2.88 & 2 & $\ldots$ & 6150 & 40 & $\ldots$ & $\ldots$ & $\ldots$ & 140 & $\ldots$ & $\ldots$ & $\ldots$ & 22.5 & $\ldots$ & $\ldots$ & $\ldots$ & 1.5 & 22.5 & 1.5 & 2.12 & 0.04 \\
\hline $\mathrm{CD}-71^{\circ} 1234$ & -2.50 & 3 & -2.60 & 6190 & 30 & $\ldots$ & 140 & 110 & 125 & $\ldots$ & 25.6 & 26.8 & 25.5 & $\ldots$ & 1.5 & 1.9 & 1.6 & 25.9 & 0.9 & 2.20 & 0.02 \\
\hline $\mathrm{BD}+26^{\circ} 3578$ & -2.54 & 2 & -2.24 & 6150 & 40 & $\ldots$ & 185 & $\ldots$ & $\ldots$ & $\ldots$ & 24.6 & $\ldots$ & $\ldots$ & $\ldots$ & 1.1 & $\ldots$ & $\ldots$ & 24.6 & 1.1 & 2.15 & 0.03 \\
\hline G186-26 & -2.85 & 2 & -2.62 & 6180 & 40 & $\ldots$ & $\ldots$ & $\ldots$ & $\ldots$ & $\ldots$ & $\ldots$ & $\ldots$ & $\ldots$ & $\ldots$ & $\ldots$ & $\ldots$ & $\ldots$ & $\ldots$ & $\ldots$ & $\ldots$ & $\ldots$ \\
\hline LP $635-14$ & -2.65 & 3 & -2.66 & 6270 & 30 & $\ldots$ & 135 & $\ldots$ & 115 & $\ldots$ & 19.1 & $\ldots$ & 21.8 & $\ldots$ & 1.5 & $\ldots$ & 1.8 & 20.2 & 1.2 & 2.15 & 0.03 \\
\hline LP $815-43$ & -3.05 & 3 & -3.00 & 6340 & 30 & 85 & 95 & $\ldots$ & $\ldots$ & 14.0 & 17.8 & $\ldots$ & $\ldots$ & 2.4 & 2.2 & $\ldots$ & $\ldots$ & 16.1 & 1.6 & 2.09 & 0.04 \\
\hline CS 22943-095 & -2.55 & 4 & -2.20 & 6140 & 40 & 95 & 75 & $\ldots$ & 110 & 20.1 & 26.8 & $\ldots$ & 23.5 & 2.2 & 2.7 & $\ldots$ & 1.9 & 23.0 & 1.3 & 2.12 & 0.03 \\
\hline $\mathrm{CD}-35^{\circ} 14849$ & -2.63 & 1 & -2.38 & 6060 & 20 & $\ldots$ & 145 & $\ldots$ & $\ldots$ & $\ldots$ & 28.8 & $\ldots$ & $\ldots$ & $\ldots$ & 1.4 & $\ldots$ & $\ldots$ & 28.8 & 1.4 & 2.17 & 0.02 \\
\hline G126-52 & -2.57 & 2 & -2.45 & 6210 & 40 & $\ldots$ & 130 & $\ldots$ & $\ldots$ & $\ldots$ & 19.1 & $\ldots$ & $\ldots$ & $\ldots$ & 1.6 & $\ldots$ & $\ldots$ & 19.1 & 1.6 & 2.08 & 0.04 \\
\hline $\mathrm{CD}-24^{\circ} 17504$ & -3.55 & 3 & -3.24 & 6070 & 20 & 85 & 90 & $\ldots$ & 95 & 19.2 & 15.1 & $\ldots$ & 19.9 & 2.4 & 2.3 & $\ldots$ & 2.2 & 18.1 & 1.3 & 1.97 & 0.03 \\
\hline \multicolumn{22}{|l|}{ Standard Stars } \\
\hline HD 74000 & -2.02 & 2 & $\ldots$ & 6040 & 30 & $\ldots$ & $\ldots$ & 130 & $\ldots$ & $\ldots$ & $\ldots$ & 22.1 & $\ldots$ & $\ldots$ & $\ldots$ & 1.6 & $\ldots$ & 22.1 & 1.6 & 2.04 & 0.04 \\
\hline HD 140283 & -2.60 & $\ldots$ & $\ldots$ & $\ldots$ & $\ldots$ & $\ldots$ & 300 & $\ldots$ & 250 & $\ldots$ & 47.7 & $\ldots$ & 48.2 & $\ldots$ & 0.7 & $\ldots$ & 0.8 & 47.9 & 0.5 & $\ldots$ & $\ldots$ \\
\hline HD 160617 & $\ldots$ & $\ldots$ & $\ldots$ & $\ldots$ & $\ldots$ & $\cdots$ & $\ldots$ & 165 & $\ldots$ & $\ldots$ & $\ldots$ & 38.1 & $\ldots$ & $\ldots$ & $\ldots$ & 1.2 & $\ldots$ & 38.1 & 1.2 & $\ldots$ & $\ldots$ \\
\hline
\end{tabular}

${ }^{a}$ WHT service observation from November 6, 1998 .

REFERENCES.-(1) Ryan \& Norris 1991; (2) Carney et al. 1994; (3) Ryan et al. 1991 adjusted to $A(\mathrm{Fe})_{\odot}=7.50$; (4) Beers et al. 1992. 


\subsubsection{Equivalent Width Calculations}

The Li $6707 \AA$ doublet separation is quite large $(0.15 \AA)$, so for the narrow range of line strengths in our program stars, the FWHM of the spectral feature is not very sensitive to the instrumental resolution, which was in any event constant throughout the observing program. We also confirmed that the line broadening of the $\mathrm{Mg} b$ lines was similar in all objects, as a check against rotational broadening or the presence of a barely resolved spectrum of a secondary companion. As a result of the similarity of our program stars, the actual width of the doublet line is expected to be constant for all of them, with only the line depth responding to equivalent width differences.

Once the continuum was defined, equivalent width measurements were made in two ways. The first measurement technique was to centroid on the $\mathrm{Li}$ doublet and then compute the equivalent width from the residual flux summed within a band $\pm 0.34 \AA$ of that centroid. The width of this band was set considering the known width of the doublet and the resolving power of the spectrograph and confirming on the spectra that this was a sensible choice. The very high $\mathrm{S} / \mathrm{N}$ observations of HD 140283, whose $\mathrm{Li}$ equivalent width $(48 \mathrm{m \AA})$ is larger than the program stars and thus sets an upper limit on the FWHM of the doublet in our (hotter) stars, showed that less than $1 \%$ of the absorbed flux would be missed over a band width of \pm 0.34 $\AA$. At the same time we avoid unwanted sensitivity to noise fluctuations that would arise if we summed over more pixels than necessary.

The second technique involved performing a Gaussian fit, but with the Gaussian FWHM fixed at $0.305 \AA$, again determined from the HD 140283 observations. As noted above, since the Li line is weak and the doublet resolved, its FWHM is determined by the doublet separation and the instrumental profile rather than the equivalent width. This procedure was adopted to avoid having noise in the line cause unphysical line widths in the fit.

Measurement of a given spectrum with the two equivalent width techniques (direct summation and constrained Gaussian fitting) showed good agreement. For the 1997 data, the mean difference between measurements and its standard deviation was $\left\langle W_{\text {sum }}-W_{\text {Gauss }}\right\rangle=-0.3 \mathrm{~m} \AA$, with $\sigma=1.7 \mathrm{m \AA}$. Similarly, for the 1996 data, the mean difference was $\left\langle W_{\text {sum }}-W_{\text {Gauss }}\right\rangle=-0.1 \mathrm{~m} \AA$, with $\sigma=1.8 \mathrm{~m} \AA$. This gives us confidence that the two techniques introduce no significant systematic differences. (This test was not repeated in 1998, since there had been no changes to the procedures.)

As noted above, two authors reduced the data independently. Once we were satisfied that both equivalent width measurement techniques gave consistent results, one approach was applied by one author to his spectral reductions, and the second technique was applied by the other. The average of the two measurements was then adopted for each epoch.

\subsubsection{Internal Errors}

Our error estimates are based on the random noise accumulated over the width of the line (e.g., Cayrel 1988). For our pixel spacing and the width over which we measure the line in the direct summation method, we obtain the relationship $\sigma(W)=184 /\left(\mathrm{S} / \mathrm{N}_{50}\right) \mathrm{m} \AA$, where $\mathrm{S} / \mathrm{N}_{50}$ is for a $50 \mathrm{~m} \AA$ pixel. Before utilizing this model in the abundance analysis, however, we made three checks for consistency.
The first test assesses whether different authors using different data reduction algorithms and software generated mutually consistent reduced spectra. The differences of equivalent widths measured by a given technique for author A's spectra and author B's spectra were $\left\langle W_{A}-W_{B}\right\rangle=$ $+0.4 \mathrm{~m} \AA$, with $\sigma=1.3 \mathrm{~m} \AA$ for the 1997 data, and $\left\langle W_{A}-W_{B}\right\rangle=-0.3 \mathrm{~m} \AA$, with $\sigma=2.6 \mathrm{~m} \AA$ for the 1996 data. The systematic differences are negligible, and the standard deviations are acceptable, being comparable with the expected noise. (The test sequence was not repeated with 1998 data.)

The second comparison investigates whether the net effect of using separate reduction routes and two distinct measurement techniques is consistent with the noise model. The error distribution inferred from the difference between each pair of measurements should be narrower than that based on photon noise, since the techniques differ in the way they measure a noisy spectrum but sample the same data and thus are exposed to the same noise. The measurement pairs can therefore be inspected to see whether they provide evidence that the random noise model is optimistic. The null hypothesis is that the error distribution inferred from the measurement pairs is not wider than that calculated from the model. The error distribution of each pair of measurements was estimated as the sample standard deviation $s_{w}=1 / \sqrt{2}\left|W_{\text {Gauss }}-W_{\text {sum }}\right|$, and a standardized statistic $Z_{\text {pair }}$ was computed by dividing by the model error, $\sigma_{W}$. Only seven of the 40 pairs have $Z_{\text {pair }}$ values exceeding 1.0 , the maximum value being 1.8 , so the null hypothesis could not be rejected. That is, we sought and failed to find evidence that the noise model is optimistic and should not be trusted.

The third test was to compare equivalent widths we measured from spectra obtained on more than one epoch to check for repeatability. We began by computing the variance $^{-1}$-weighted mean equivalent width for each star, $\bar{W}$, and the variance of the weighted mean, $\sigma_{\bar{W}}$ (e.g., Bevington 1969). These values are given in Table 2. Next we computed the standardized residual, $Z$, for each observation as $Z=(W-\bar{W}) / \sigma_{W}$. The $Z$-distribution has a standard deviation of 1.0 (or 1.1 if restricted to stars with three observations), and the standardized residual with the largest magnitude is +2.1 , which shows that all but one of the 30 values falls within \pm 2 standard deviations of the mean. In other words, the repeatability achieved from run to run is again consistent with the noise model.

\subsubsection{External Errors}

Although it is internal consistency that is most important for this study, it is nevertheless valuable to know whether or not our data are consistent with the work of others. ${ }^{5}$ Norris et al. (1994) and Ryan (1995) highlighted differences between Li equivalent width measurements for LP 815-43, which ranged over a factor of 2 from $13 \pm 2 \mathrm{m \AA}$ and $15 \pm 3 \mathrm{m \AA}$ (Norris et al. 1994) to $22 \pm 2.1 \mathrm{m \AA}$ (Thorburn 1994) and $27 \pm(3-6) \mathrm{mA}$ (Spite \& Spite 1993). Our new measurement, $16.1 \pm 1.6 \mathrm{m \AA}$, is consistent with our earlier measurements and reemphasizes the importance of homogeneity in obtaining small random errors. It is the development of a large homogeneous data set in the current work that has allowed

\footnotetext{
${ }^{5}$ We elected not to risk decreasing the homogeneity of the data set by combining it with other studies from the literature, including our own earlier work.
} 
us to probe the scatter about the Li plateau with a much higher precision than previous work, typically conducted at the 0.06-0.10 dex level.

Most studies in which new Li data have been presented have analyzed only a dozen or so stars, so it is difficult to establish what, if any, systematic differences exist for the various studies, since the systematics can differ from one study to the next. Ryan et al. (1996a) addressed this issue using the extensive data set of Thorburn (1994) as a baseline for comparison but found "either there were too few stars for a reliable comparison, or else the differences that existed could not confidently be ascribed to systematic errors amenable to transformation" onto a unified system. The one exception was a small but clearly systematic offset (3 mA) for the Thorburn versus Spite \& Spite (1993) samples.

In Table 3 we present previous Li equivalent width measurements of our program stars and the three "standard" stars. Perusal of the list shows no cause for alarm that our data are systematically different from our previous work or that of others, except perhaps for the Spite \& Spite (1993) sample, as discussed above. The most precise observations in the table are the high $\mathrm{S} / \mathrm{N}$, high resolving power data obtained by Smith, Lambert, \& Nissen (1998) (using different facilities from ours) to measure the ${ }^{6} \mathrm{Li} /{ }^{7} \mathrm{Li}$ isotope ratio. We plot our measurements against theirs in Figure 2. For the five stars in common, two agree within $1 \sigma$, and the remaining three agree within $1.2-1.8 \sigma$. The mean difference $\langle R N B-S L N\rangle=-0.5 \mathrm{m \AA}$, with sample standard deviation $1.6 \mathrm{~m} \AA$. This comparison leaves us confident that, even though it is the high internal precision that is required for this study, our equivalent width measurements are also of high absolute accuracy.

\subsection{Radial Velocity Measurements}

To provide a check on unrecognized binarity among our program stars, we have also measured precise radial veloci-

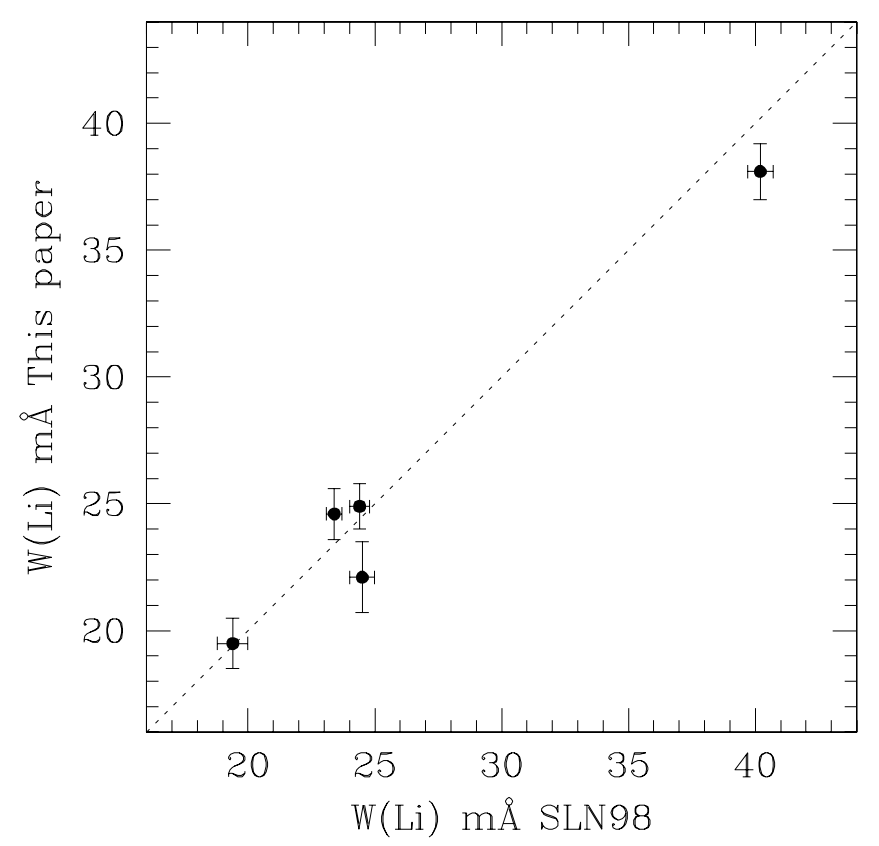

Fig. 2.-Comparison of equivalent width measurements for stars in common to our work and the accurate isotope ratio work of Smith et al. (1998). Our data are in agreement with theirs within $\pm 1.8 \sigma$ at worst, and considerably better in many cases. The dotted line is the $1: 1$ locus.
TABLE 3

EXTERNAL COMPARISON WITH LITERATURE DATA

\begin{tabular}{|c|c|c|c|}
\hline Star & $W_{\mathrm{Li}}$ & $\sigma_{W}$ & References \\
\hline LP $651-4 \ldots \ldots \ldots \ldots$ & 19.6 & 1.6 & RNB99 \\
\hline \multirow[t]{3}{*}{ G4-37 ............. } & 25.9 & 2.3 & RNB99 \\
\hline & 20.0 & 2.8 & HT91 \\
\hline & 19.0 & 3.4 & T94 \\
\hline \multirow[t]{3}{*}{ LP $831-70 \ldots \ldots \ldots$} & 23.1 & 1.4 & RNB99 \\
\hline & 26.0 & 2.1 & SS93 \\
\hline & 23.0 & 2.6 & T94 \\
\hline \multirow[t]{4}{*}{$\mathrm{CD}-33^{\circ} 1173 \ldots \ldots$} & 17.2 & 1.2 & RNB99 \\
\hline & 17.0 & 2.7 & SS93 \\
\hline & 12.0 & 2.4 & T94 \\
\hline & 10.0 & 1.5 & NRS94 \\
\hline \multirow[t]{9}{*}{$\mathrm{BD}+03^{\circ} 740 \ldots \ldots$} & 19.5 & 1.1 & RNB99 \\
\hline & 17.0 & 1.5 & HP88 \\
\hline & 21.0 & 4.8 & RMB88 \\
\hline & 21.0 & 3.7 & ТВ93 \\
\hline & 19.3 & 1.0 & HT94 \\
\hline & 24.0 & 2.4 & T94 \\
\hline & 23.0 & 1.0 & RBDT96 \\
\hline & 25.0 & 4.5 & SFNS93 \\
\hline & 19.4 & 0.6 & SLN98 \\
\hline \multirow{3}{*}{$\mathrm{BD}+24^{\circ} 1676 \ldots \ldots$} & 21.1 & 2.2 & RNB99 \\
\hline & 26.0 & 2.2 & HT91 \\
\hline & 28.0 & 2.9 & T94 \\
\hline \multirow[t]{2}{*}{$\mathrm{BD}+20^{\circ} 2030 \ldots \ldots$} & 20.5 & 2.0 & RNB99 \\
\hline & 23.0 & 2.2 & T94 \\
\hline \multirow[t]{3}{*}{$\mathrm{BD}+09^{\circ} 2190 \ldots \ldots$} & 14.7 & 1.3 & RNB99 \\
\hline & 18.0 & 3.4 & T94 \\
\hline & 20.0 & 1.5 & RBDT96 \\
\hline \multirow{3}{*}{$\mathrm{BD}+1^{\circ} 2341 \mathrm{p} \ldots \ldots$} & 17.8 & 1.6 & RNB99 \\
\hline & 23.0 & 4.5 & HD87 \\
\hline & 21.0 & 2.6 & T94 \\
\hline \multirow[t]{11}{*}{ HD 84937} & 24.9 & 1.1 & RNB99 \\
\hline & 18.0 & 2.2 & SS82 \\
\hline & 23.0 & 1.0 & B85 \\
\hline & 20.0 & 3.0 & HD87 \\
\hline & 25.0 & 1.0 & PHD89 \\
\hline & 24.5 & 1.0 & НТ94 \\
\hline & 22.0 & 2.5 & T94 \\
\hline & 25.0 & 1.0 & RBDT96 \\
\hline & 26.2 & 1.0 & RBDT96 \\
\hline & 25.0 & & SLN93 \\
\hline & 24.4 & 0.4 & SLN98 \\
\hline \multirow[t]{3}{*}{$\mathrm{BD}-13^{\circ} 3442 \ldots \ldots$} & 21.0 & 1.4 & RNB99 \\
\hline & 30.0 & 3.4 & T94 \\
\hline & 19.0 & 1.0 & RBDT96 \\
\hline \multirow[t]{6}{*}{ G64-12 …........ } & 21.2 & 1.1 & RNB99 \\
\hline & 25.0 & 5.0 & SSPC87 \\
\hline & 23.0 & 5.0 & RBM87 \\
\hline & 31.0 & $4.0 ?$ & SS93 \\
\hline & 28.0 & 3.5 & TB93 \\
\hline & 28.0 & 3.6 & T94 \\
\hline \multirow[t]{5}{*}{ G64-37 ............ } & 18.2 & 1.5 & RNB99 \\
\hline & 14.0 & 1.6 & T94 \\
\hline & 14.0 & 2.0 & NRS94 \\
\hline & 16.0 & 1.5 & RBDT96 \\
\hline & 18.0 & 4.5 & SFNS96 \\
\hline \multirow[t]{2}{*}{$\mathrm{BD}+26^{\circ} 2621 \ldots \ldots$} & 22.5 & 1.5 & RNB99 \\
\hline & 20.0 & 2.9 & T94 \\
\hline \multirow[t]{2}{*}{$\mathrm{CD}-71^{\circ} 1234 \ldots \ldots$} & 25.9 & 0.9 & RNB99 \\
\hline & 27.0 & 2.9 & T94 \\
\hline $\mathrm{BD}+26^{\circ} 3578 \ldots \ldots$ & 24.6 & 1.1 & RNB99 \\
\hline & 24.0 & 1.7 & SMS84 \\
\hline & 24.0 & 1.5 & HD87 \\
\hline & 22.2 & 1.0 & HT94 \\
\hline & 23.4 & 0.3 & SLN98 \\
\hline
\end{tabular}


TABLE 3-Continued

\begin{tabular}{|c|c|c|c|}
\hline Star & $W_{\mathrm{Li}}$ & $\sigma_{W}$ & References \\
\hline \multirow[t]{2}{*}{ LP $635-14 \ldots \ldots \ldots$} & 20.2 & 1.2 & RNB99 \\
\hline & 24.0 & 3.9 & T94 \\
\hline \multirow[t]{5}{*}{ LP $815-43 \ldots \ldots \ldots$} & 16.1 & 1.6 & RNB99 \\
\hline & 27.0 & 2.2 & SS93 \\
\hline & 22.0 & 2.1 & T94 \\
\hline & 15.0 & 3.2 & NRS94 \\
\hline & 13.0 & 1.6 & NRS94 \\
\hline CS $22943-095 \ldots \ldots$ & 23.0 & 1.3 & RNB99 \\
\hline \multirow[t]{2}{*}{$-35^{\circ} 14849 \ldots \ldots \ldots \ldots$} & 28.8 & 1.4 & RNB99 \\
\hline & 30.8 & 5.0 & SFNS96 \\
\hline \multirow[t]{2}{*}{ G126-52 ........... } & 19.1 & 1.6 & RNB99 \\
\hline & 26.0 & 3.6 & T94 \\
\hline \multirow[t]{4}{*}{$\mathrm{CD}-24^{\circ} 17504 \ldots \ldots$} & 18.1 & 1.3 & RNB99 \\
\hline & 22.0 & 4.1 & SS93 \\
\hline & 21.0 & 3.4 & T94 \\
\hline & 19.0 & 2.3 & NRS94 \\
\hline \multirow[t]{4}{*}{ HD 74000 . } & 22.1 & 1.6 & RNB99 \\
\hline & 25.0 & 3.4 & SS86 \\
\hline & 24.5 & 0.9 & HT97 \\
\hline & 24.5 & 0.5 & SLN98 \\
\hline \multirow[t]{5}{*}{ HD $140283 \ldots \ldots \ldots$} & 47.9 & 0.5 & RNB99 \\
\hline & 50.0 & $\ldots$ & RMB88 \\
\hline & 46.0 & 1.8 & T94 \\
\hline & 48.0 & 0.8 & NRS94 \\
\hline & 46.0 & 3.0 & SFNS96 \\
\hline \multirow[t]{3}{*}{ HD $160617 \ldots \ldots \ldots$} & 38.1 & 1.2 & RNB99 \\
\hline & 43.0 & 3.0 & SFNS96 \\
\hline & 40.2 & 0.5 & SLN98 \\
\hline
\end{tabular}

REFERENCES.-SS82, Spite \& Spite 1982; SMS84 Spite, Maillard, \& Spite 1984; B85, Boesgaard 1985; SS86, Spite \& Spite 1986; SSPC87, Spite et al. 1987; HD87, Hobbs \& Duncan 1987; HP88, Hobbs \& Pilachowski 1988; RMB88, Rebolo, Molaro, \& Beckman 1988; PHD89, Pilachowski, Hobbs, \& De Young 1989; HT91, Hobbs \& Thorburn 1991; SS93, Spite \& Spite 1993; TB93, Thorburn \& Beers 1993; HT94, Hobbs \& Thorburn 1994; T94, Thorburn 1994; NRS94, Norris et al. 1994; RBDT96, Ryan et al. 1996a; SLN93, Smith et al. 1993; SFNS96, Spite et al. 1996; HT97, Hobbs \& Thorburn 1997; SLN98, Smith et al. 1998; RNB99, this work.

ties. Although there are few spectral lines near Li 6707, the echelle spectra extend sufficiently blueward to include the $\mathrm{Mg} b$ triplet and neighboring lines. The spectra were crosscorrelated over the wavelength region 5160-5200 A, using the 1997 observation of HD 140283 as the template. The zero-point velocity was then set by measurements of 42 apparently unblended lines in that spectrum, which gave a formal error of $\pm 0.1 \mathrm{~km} \mathrm{~s}^{-1}$ (1 standard error).

The heliocentric radial velocity for each epoch is given in Table 4, along with measurements from Carney et al. (1994, CLLA94 in the table). The "Notes" column gives, for the Carney et al. entries, the dispersion $(1 \sigma)$ of their velocity measurements, the number of observations made, and the span (in days) of their series of observations. Excluding the previously known single-lined spectroscopic binary (SB1), $\mathrm{BD}+20^{\circ} 2030$, and one clear new detection in this work, $\mathrm{CD}-71^{\circ} 1234$, the typical scatter for our multiple measurements and for the difference between our measurements and those of Carney et al. is $0.3 \mathrm{~km} \mathrm{~s}^{-1}(1 \sigma)$. This is consistent with the external accuracy we have obtained previously with similar observational material (Norris, Ryan, \& Beers 1997). There is no overwhelming evidence for binarity in the other stars at this level of accuracy; unrecognized binaries
TABLE 4

Heliocentric Radial Velocities for Program Stars

\begin{tabular}{|c|c|c|c|}
\hline Star & $\begin{array}{c}v_{\mathrm{rad}} \\
\left(\mathrm{km} \mathrm{s}^{-1}\right)\end{array}$ & References & Notes \\
\hline \multirow[t]{3}{*}{ LP $651-4 \ldots \ldots \ldots \ldots$} & 12.6 & 96 & \\
\hline & 12.2 & 97 & \\
\hline & 12.3 & $98 \mathrm{~B}$ & \\
\hline \multirow[t]{2}{*}{ G4-37 .............. } & -108.5 & CLLA94 & $1.0,41,3633$ \\
\hline & -108.5 & 97 & \\
\hline \multirow[t]{2}{*}{ LP $831-70 \ldots \ldots \ldots$} & -48.1 & 97 & \\
\hline & -48.4 & $98 \mathrm{~B}$ & \\
\hline \multirow[t]{2}{*}{$\mathrm{CD}-33^{\circ} 1173 \ldots \ldots$} & 47.4 & 97 & \\
\hline & 47.0 & $98 \mathrm{~B}$ & \\
\hline \multirow[t]{3}{*}{$\mathrm{BD}+3^{\circ} 740 \ldots \ldots \ldots$} & 173.8 & CLLA94 & $0.9,14,1809$ \\
\hline & 173.7 & $98 \mathrm{~A}$ & \\
\hline & 173.7 & 98B & \\
\hline \multirow{2}{*}{$\mathrm{BD}+24^{\circ} 1676 \ldots \ldots$} & -238.4 & CLLA94 & $1.0,17,2210$ \\
\hline & -238.0 & $98 \mathrm{~A}$ & \\
\hline \multirow[t]{2}{*}{$\mathrm{BD}+20^{\circ} 2030 \ldots \ldots$} & -67.2 & CLLA94 & $0.9,34,382$, SB1 \\
\hline & -55.9 & $98 \mathrm{~A}$ & \\
\hline \multirow[t]{3}{*}{$\mathrm{BD}+9^{\circ} 2190 \ldots \ldots \ldots$} & 266.1 & CLLA94 & $1.5,38,2839$ \\
\hline & 266.1 & $98 \mathrm{~A}$ & \\
\hline & 265.8 & WHT98 & \\
\hline \multirow{2}{*}{ G48-29 ............. } & -57.4 & CLLA94 & $1.6,31,2952$ \\
\hline & -57.2 & $98 \mathrm{~A}$ & \\
\hline \multirow[t]{2}{*}{ HD $84937 \ldots \ldots \ldots \ldots$} & -14.8 & CLLA94 & $0.9,35,3572$ \\
\hline & -15.0 & $98 \mathrm{~A}$ & \\
\hline \multirow[t]{2}{*}{$\mathrm{BD}-13^{\circ} 3442 \ldots \ldots$} & 115.8 & $98 \mathrm{~A}$ & \\
\hline & 115.2 & $98 \mathrm{~B}$ & \\
\hline \multirow[t]{3}{*}{ G64-12 ............. } & 441.9 & CLLA94 & $1.7,24,4406$ \\
\hline & 441.8 & 97 & \\
\hline & 442.2 & $98 \mathrm{~B}$ & \\
\hline \multirow[t]{3}{*}{ G64-37 } & 81.2 & CLLA94 & $1.4,18,1234$ \\
\hline & 80.7 & 97 & \\
\hline & 81.6 & $98 \mathrm{~A}$ & \\
\hline \multirow[t]{2}{*}{ G166-54 ............. } & -62.9 & CLLA94 & $1.2,18,1508$ \\
\hline & -62.9 & $98 \mathrm{~B}$ & \\
\hline \multirow[t]{3}{*}{$\mathrm{CD}-71^{\circ} 1234 \ldots \ldots$} & 231.0 & 97 & \\
\hline & 220.9 & $98 \mathrm{~A}$ & \\
\hline & 215.0 & $98 \mathrm{~B}$ & New SB1 \\
\hline \multirow[t]{2}{*}{$\mathrm{BD}+26^{\circ} 3578 \ldots \ldots$} & -129.1 & CLLA94 & $0.7,17,3072$ \\
\hline & -129.4 & 97 & \\
\hline \multirow{2}{*}{ LP $635-14 \ldots \ldots \ldots$} & -117.9 & 97 & \\
\hline & -117.0 & 98B & \\
\hline \multirow[t]{2}{*}{ LP $815-43 \ldots \ldots \ldots$} & -4.1 & 96 & \\
\hline & -4.2 & 97 & \\
\hline CS $22943-095 \ldots \ldots$ & -150.1 & 96 & \\
\hline & -150.7 & 97 & \\
\hline & -150.5 & $98 \mathrm{~B}$ & \\
\hline $\mathrm{CD}-35^{\circ} 14849 \ldots \ldots$ & 108.0 & 97 & \\
\hline G126-52 ........... & -242.1 & CLLA94 & $1.2,21,1799$ \\
\hline & -241.4 & 97 & \\
\hline BD $-24^{\circ} 17504 \ldots \ldots$ & 135.8 & 96 & \\
\hline & 135.7 & 97 & \\
\hline & 135.5 & 98B & \\
\hline & Standa & Stars & \\
\hline HD $74000 \ldots \ldots \ldots \ldots$ & 206.3 & CLLA94 & $0.9,41,3299$ \\
\hline & 205.9 & $98 \mathrm{~A}$ & \\
\hline HD $140283 \ldots \ldots \ldots$ & -170.9 & CLLA94 & $0.8,19,3115$ \\
\hline & -171.1 & 97 & \\
\hline & -170.4 & $98 \mathrm{~B}$ & \\
\hline HD $160617 \ldots \ldots \ldots$ & 99.4 & $98 \mathrm{~A}$ & \\
\hline
\end{tabular}


must have very low velocity amplitudes and/or very long periods, which, statistically at least, suggests that their companions will have minimal impact on our analysis. Consequently, we may infer that the impact of unrecognized binarity is minor.

\section{EFFECTIVE TEMPERATURES}

\subsection{Observational Indices}

Deliyannis et al. (1993) attempted to circumvent the uncertainties in color-temperature transformations by working with color alone. However, it is implicit in such a procedure that the color, along with its random errors, accurately ranks the stars over the full range of the sample. We have taken a different approach to minimize the effects of errors, that of restricting the diversity of stellar types at sample selection. The dereddened $c_{1}^{0}$ versus $(b-y)_{0}$ diagram (Fig. 3) confirms that the stars are within $0.05 \mathrm{mag}$ in $b-y$ of the Population II main-sequence turnoff. Nevertheless, to reach the desired level of accuracy we need to resolve even small temperature differences between almost identical stars and hence fully utilize the available temperature indices (e.g., Spite et al. 1996). We can improve on the studies that adopt only a single color by having up to six indices $(b-y$, $B-V, V-R, R-I, \beta$, and HP2) on which to base effective temperatures; these are shown in Figure 4 as a function of $(b-y)_{0}$.

Several color-effective temperature scales from the literature are also shown in Figure 4. Figure $4 a$ shows the $B-V$ versus $b-y$ theoretical colors of Bell \& Oke (1986) for $[\mathrm{Fe} / \mathrm{H}]=-2$ and $\lg g=4.0$, which coincide roughly with the data, and the transformation of Magain (1987, that paper's eqs. [15] and [16]) at $[\mathrm{Fe} / \mathrm{H}]=-2.8$ (the mean metallicity of our sample), which sits away from the data, showing that Magain's $B-V$ and $b-y$ scales are not mutually consistent for these stars. Magain's and Bell \& Oke's $b-y$ scales are almost identical for metal-poor turnoff stars, Bell \& Oke's scale being hotter by $7 \mathrm{~K}$ at

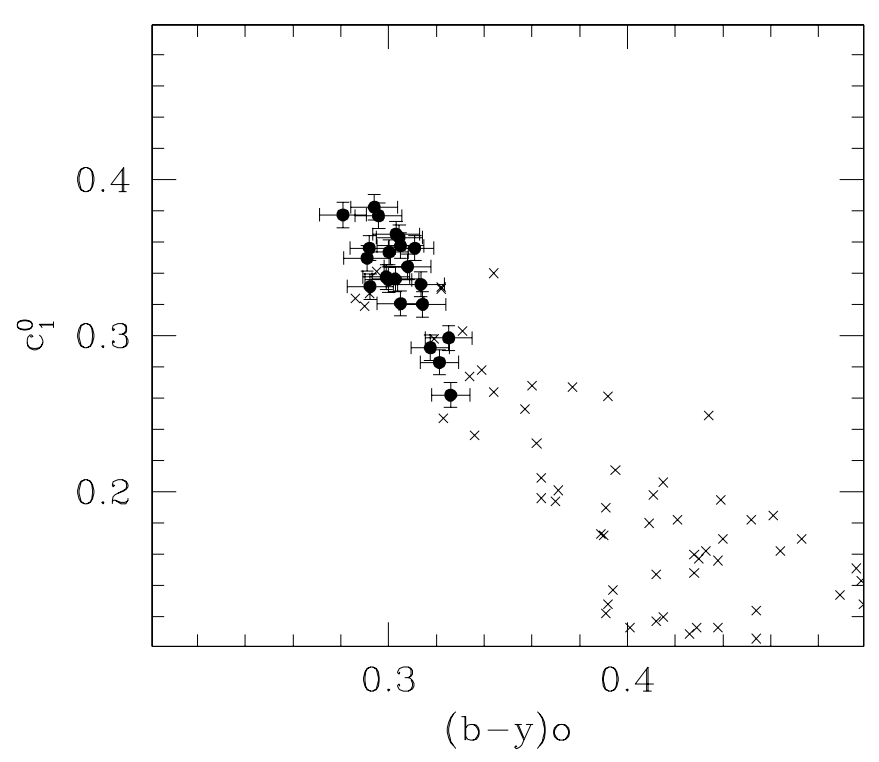

FIG. 3.-Dereddened Strömgren $c_{1}^{0}$ vs. $(b-y)_{0}$ diagram showing our turnoff sample (solid symbols) against the general halo sample with $[\mathrm{Fe} / \mathrm{H}]<-1.0$ of Schuster, Parrao, \& Contreras Martinez (1993; crosses).

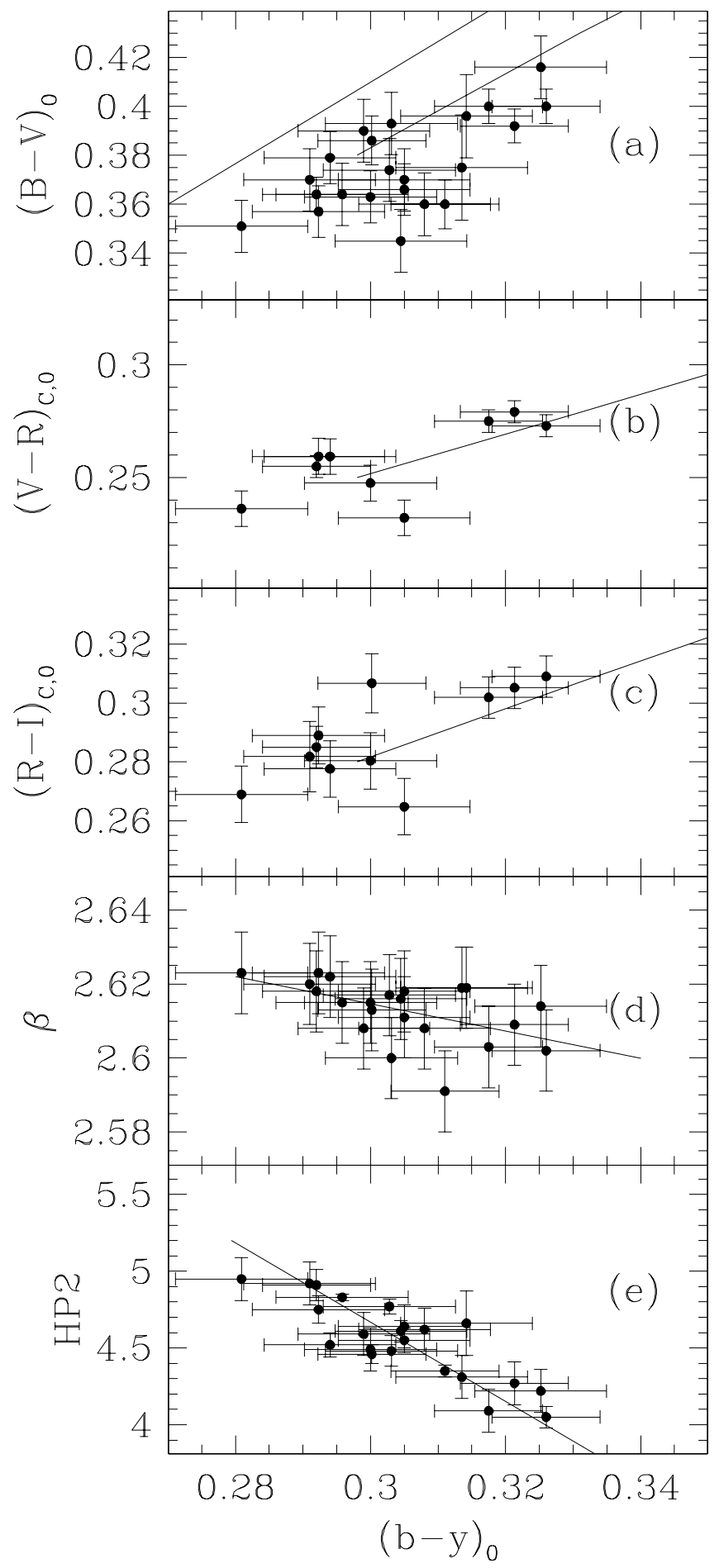

Fig. 4.-Dereddened indices available to measure effective temperature, as a function of $(b-y)_{0}$. (a) The solid line coinciding with the data is from the theoretical colors of Bell \& Oke (1986) for $[\mathrm{Fe} / \mathrm{H}]=-2$ and $\lg g=4.0$. The solid line sitting away from the data is the transformation of Magain (1987) at $[\mathrm{Fe} / \mathrm{H}]=-2.8 .(b, c)$ The solid lines show the Bell \& Oke theoretical colors. $(d, e)$ The solid lines show the least-squares fits of $(b-y)_{0}$ to the index, used to predict $(b-y)_{0}$ from the measured indices.

$6100 \mathrm{~K}$ and $22 \mathrm{~K}$ at $6300 \mathrm{~K}$. The Bell \& Oke $(V-R)_{\mathrm{C}}$ and $(R-I)_{\mathrm{C}}$ colors are shown in Figures $4 b$ and $4 c$. Figures $4 d$ and $4 e$ show the good correlation between $(b-y)_{0}$ and the Balmer line indices, $\beta$ and especially HP2. A least-squares fit to the data permits estimates of the $(b-y)_{0}$ colors 
from the observed Balmer indices, which we call $(b-y)_{\beta}$ and $(b-y)_{\mathrm{HP} 2}$.

\subsection{Calibrations}

Because our sample spans a 1 dex range in metallicity, it is important to understand the sensitivity of the effective temperature indicators to $[\mathrm{Fe} / \mathrm{H}]$. In very metal-poor turnoff stars, we do not expect the chosen indices to be sensitive to abundance. We sought to verify this through available effective temperature calibrations and especially to check the sensitivity of $B-V$ since this index was expected to have the greatest dependence, if any.

According to Magain's (1987) $B-V$ empirical calibration, which is based on 11 stars with $[\mathrm{Fe} / \mathrm{H}]<-1$ and temperatures derived from the infrared flux method (IRFM), changing $[\mathrm{Fe} / \mathrm{H}]$ from -2.5 to -3.5 would change the inferred $T_{\text {eff }}$ of turnoff stars by less than $1 \mathrm{~K}$. However, the small size of this figure may be driven by the analytical form of Magain's fitting function, which is linear in metallicity $\left(Z / Z_{\odot}\right)$ and hence loses sensitivity to this variable at even moderate metal deficiency. It also should be borne in mind that the most metal-poor member of Magain's calibration set was HD 140283, for which $[\mathrm{Fe} / \mathrm{H}]=-2.6$ (Ryan, Norris, \& Beers 1996b), at the upper end of the present sample's metallicity range.

One recent and extensive calibration is the table of synthetic colors computed by Kurucz (1993) for a comprehensive range of observable indices, tracing metallicity sensitivity down to $[\mathrm{Fe} / \mathrm{H}]=-5$. Gratton, Carretta, \& Castelli (1996) have shown that zero-point differences are still found with other calibrations, but they nevertheless adopted the Kurucz metallicity dependence in devising their own transformation. This metallicity dependence is shown in Figures $5 a-5 e$ (solid curves) for pairs of colors typical of metal-poor turnoff stars. Magain's (1987) $B-V$ and $b-y$ calibrations are also shown (dashed curves).

A more recent empirical calibration is that by Alonso, Arribas, \& Martinez-Roger (1996a), which uses the large calibrating set of IRFM temperatures of Alonso et al. (1996b). They give fitting functions for a wide range of stellar types, but unfortunately these become nonphysical for turnoff stars below $[\mathrm{Fe} / \mathrm{H}] \sim-2.5$. Figure $5 a$ (dotted curves) shows the run of $T_{\text {eff }}$ as a function of $[\mathrm{Fe} / \mathrm{H}]$ for a pair of $B-V$ colors $(0.35$ and 0.40$)$ appropriate to turnoff stars. Although the curves exhibit a reduction in sensitivity to metal abundance as $[\mathrm{Fe} / \mathrm{H}]$ falls from -1.0 to -2.6 , the fitting function goes through a minimum and climbs again at lower metallicity. It is unreasonable to expect that stars of yet lower $[\mathrm{Fe} / \mathrm{H}]$ exhibit stronger sensitivity to metallicity, and such behavior is not supported by the Kurucz (1993) colors. Clearly this behavior reflects the form of the fitting function and the values of the coefficients rather than the characteristics of metal-poor turnoff stars. (Alonso et al.'s fitting functions have a quadratic form and so do not have the monotonically decreasing property of Magain's.) Although Alonso et al.'s $B-V$ calibration may be an improvement for the majority of stars, it is not applicable to our very metal-poor sample. Similar results obtain for most other indices in Alonso et al.'s calibrations. Figures $5 b-5 e$ show that the $V-R, b-y$, and $\beta$ calibrations also show nonphysical forms at very low metallicity, $b-y$ being the most dramatic. The most we can infer is that the metallicity sensitivity appears to saturate (reaches a minimum) once

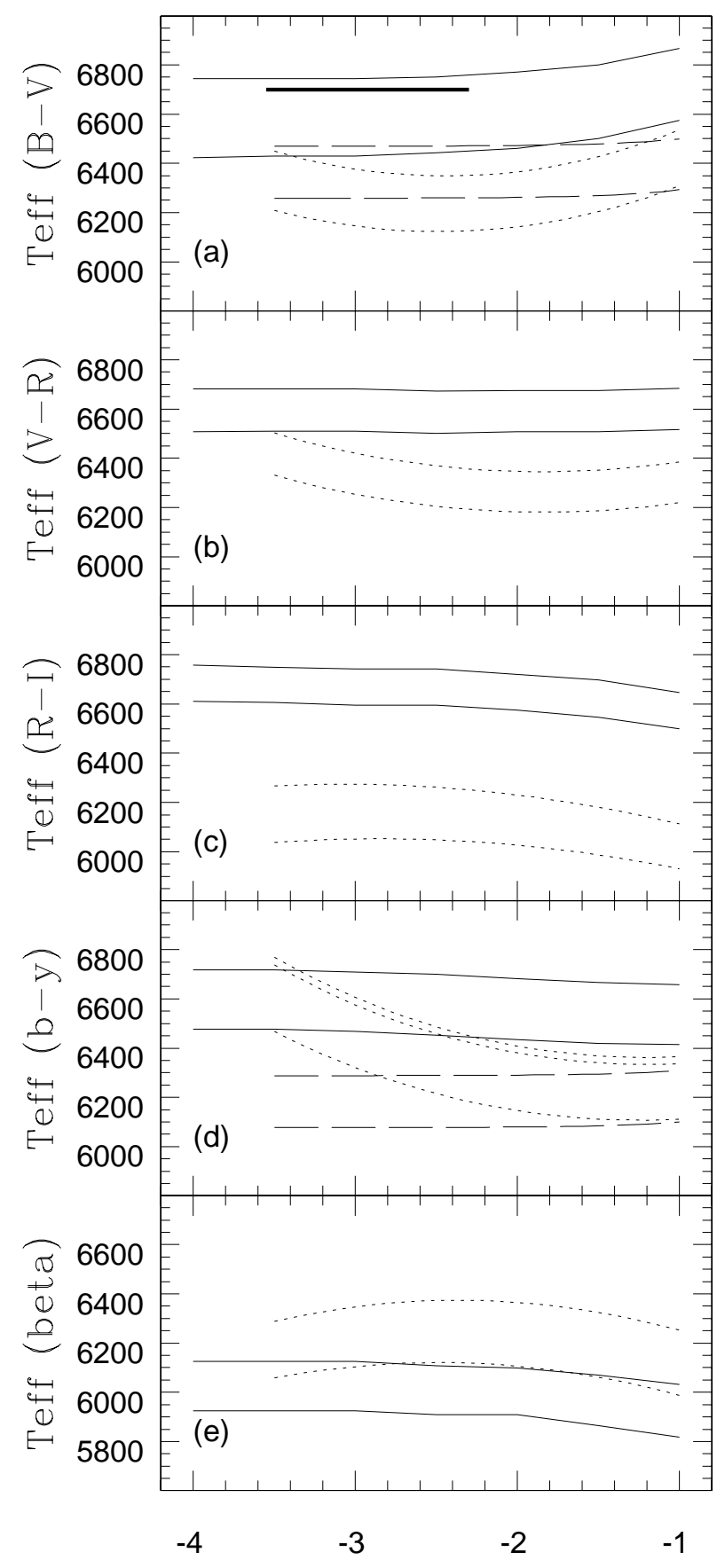

$[\mathrm{Fe} / \mathrm{H}]$

FIG. 5.-Metallicity sensitivity of color-effective temperature calibrations for metal-poor turnoff stars: synthetic colors of Kurucz (1993) (solid curve); empirical fit to IRFM temperatures by Magain (1987) [dashed curve, $(a, d)$ only]; and empirical fit to IRFM temperatures by Alonso et al. (1996a) (dotted curve). Pairs of values are traced for each color. In the Kurucz and Magain (1987) calibrations, the sensitivity to metallicity decreases as expected as $[\mathrm{Fe} / \mathrm{H}]$ falls from -1 , whereas the Alonso et al. calibrations go through a minimum before increasing nonphysically toward yet lower metallicity. (a) $B-V=0.35$ and 0.40 . The solid bar shows the metallicity range of our sample. $(b)(V-R)_{C}=0.26$ and 0.28 (which were transformed to Johnson colors for Alonso et al.'s calibration). (c) $(R-I)_{C}=0.29$ and 0.31 (which were transformed to Johnson colors for Alonso et al.'s calibration). (d) $b-y=0.29$ and 0.32 . Alonso et al.'s calibration appears highly nonphysical over the range $[\mathrm{Fe} / \mathrm{H}]<-1$. Their $b-y=0.29$ curve is shown for two values of $c_{1}=0.32$ and 0.38 ; the lower curve is for $c_{1}=0.30$. (e) $\beta=2.60$ and 2.62 for Alonso et al. calibration and $\beta=2.65$ and 2.66 for Kurucz calibration. 
$[\mathrm{Fe} / \mathrm{H}]$ falls to -2.5 and, for some indices, saturates at considerably higher $[\mathrm{Fe} / \mathrm{H}]$.

The metallicity range of our sample is indicated by a solid bar in Figure 5a. What is important for the present study is that over the metallicity range of our sample, $-3.5 \leq$ $[\mathrm{Fe} / \mathrm{H}] \leq-2.3$, all of the effective temperature indices we have used should possess essentially zero sensitivity to metallicity. In Kurucz's (1993) calibration, which is the only one of the three sensitive over our abundance regime, no index demonstrates a change by more than $18 \mathrm{~K}$ over the interval from $-3.5 \leq[\mathrm{Fe} / \mathrm{H}] \leq-2.5$. We thus feel justified in assuming that there is no significant metallicity dependence in any of our photometric indices.

We note for completeness that we inspected the data for any metallicity dependence in the difference between the dereddened $B-V$ color and that predicted from $b-y$ by Bell \& Oke's (1986) $B-V$ versus $b-y$ calibration. No significant dependence was found.

\subsection{Combining Indices}

We calculated temperatures for each of the indices shown, following Magain (1987) and Bell \& Oke (1986) in adopting linear relationships between $T_{\text {eff }}$ and color over the short temperature range involved and adopting zero sensitivity to metallicity due to the considerable metaldeficiency of our sample. Minor extrapolation was required to use the Bell \& Oke calibrations for stars hotter than $6250 \mathrm{~K}$.

Temperature scales from different indices are seldom in agreement. We went through the exercise of computing linear transformations between the different temperature scales, but given the short temperature baseline covered by our stars we doubted the reliability of the scale factors (slope coefficients) and have instead applied zero-point adjustments only. We use Magain's (1987) $b-y$ scale as the zero point (which essentially matches Bell \& Oke's $b-y$ scale) and offset the Bell \& Oke-scale temperatures as follows: $\quad T(V-R)=T(V-R)_{\mathrm{BO}}-165 \quad \mathrm{~K}, \quad T(R-I)=$ $T(R-I)_{\mathrm{BO}}-155 \mathrm{~K}$, and $T(B-V)=T(B-V)_{\mathrm{BO}}-85 \mathrm{~K}$. We also computed a temperature (on Magain's scale) based on the estimates $(b-y)_{\beta}$ and $(b-y)_{\mathrm{HP} 2}$.

The adopted effective temperature for each star is the variance $^{-1}$-weighted mean of the $b-y,(b-y)_{\beta},(b-y)_{\mathrm{HP} 2}$, and rebased $B-V, V-R$, and $R-I$ temperatures, using the variances for the individual temperature estimates determined from the photometric errors. The error estimates in temperatures derived from the $\beta$ and HP2 indices include both the uncertainty in the measurement of each spectral index itself and the uncertainty in the $(b-y)_{0}$ value that is inferred from the least-squares fit (Figs. $4 d$ and $4 e$ ). Temperatures and uncertainties are given in Table 2 (to the nearest $10 \mathrm{~K})$. Temperatures from $B-V, b-y, \beta$, and HP2 are available for almost all stars, resulting in an average over four estimates (although the errors in $T_{\beta}$ result in low weight for the $\beta$ index), with additional data from $V-R$ and $R-I$ being available for roughly half of the sample.

\section{THE OBSERVED AND INTRINSIC SPREADS IN LITHIUM ABUNDANCE}

Figure $6 a$ presents the lithium equivalent widths, $\bar{W}$, as a function of effective temperature. Because the $\mathrm{Li}$ line is weak, its equivalent width varies linearly with abundance, so $\lg \bar{W}$ is linear in logarithmic abundance. In Figure $6 b$,
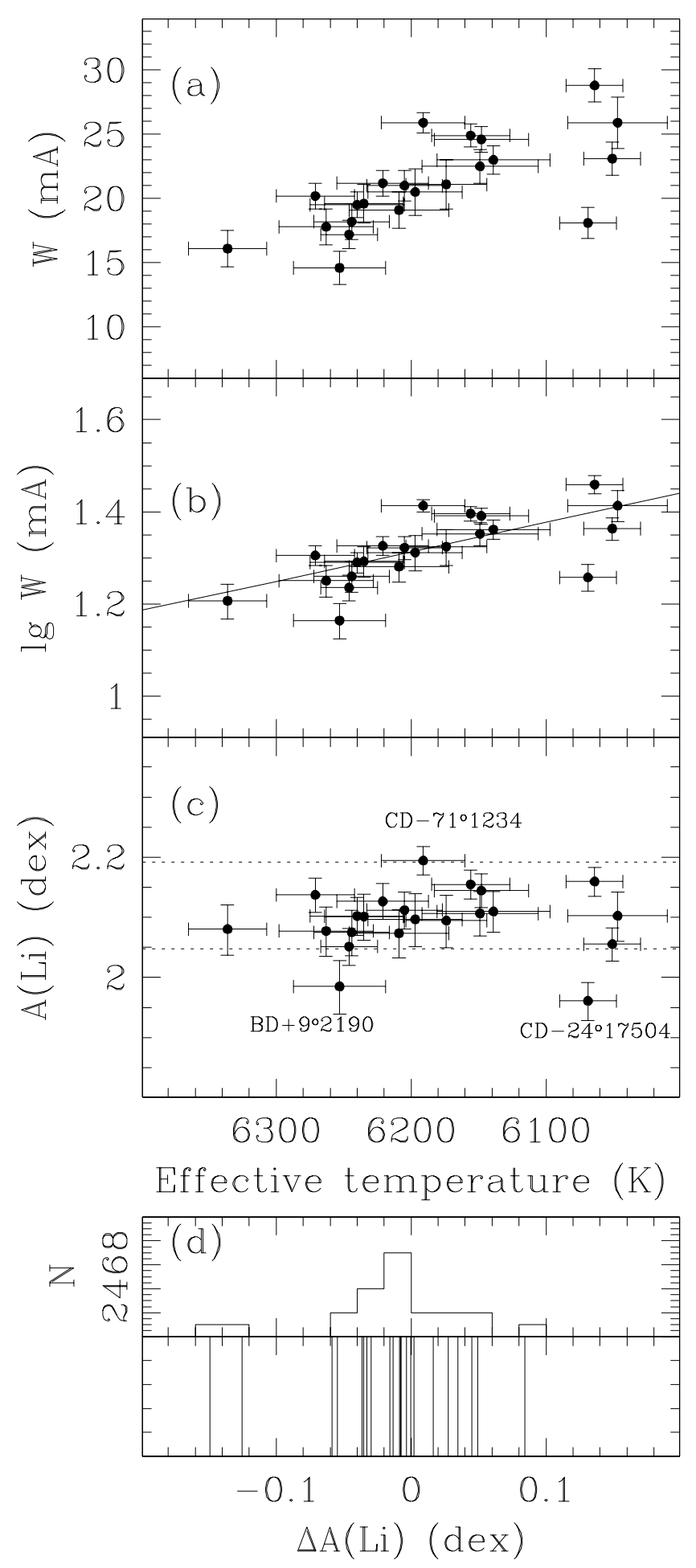

Fig. 6. - (a) Equivalent widths vs. effective temperature. (b) Equivalent widths on lg scale, which is linear in $A(\mathrm{Li})$. The solid line is for $A(\mathrm{Li})=2.11$. (c) Spread in $A(\mathrm{Li})$ about the 2.11 dex locus. The dashed lines are at \pm 0.072 dex $(2 \sigma)$ from the mean of the majority. (d) Histogram (top) and stripe plot (bottom) of $A(\mathrm{Li})$ spread. The sample is seen to consist of a well defined bell curve to which the majority of the data conform, plus two stars lower in $A(\mathrm{Li})$ by $\sim 0.14$ dex. See text for discussion.

the solid curve corresponds to the lithium abundance $A(\mathrm{Li})=2.11$, based on the computation using Bell models at $\lg g=4.0$ and $[\mathrm{Fe} / \mathrm{H}]=-2$ presented by Ryan et al. (1996a, their Table 5). In that work, synthetic spectra were 
computed for a range of input effective temperatures and lithium abundances, using R. A. Bell's (1983, private communication) dwarf model atmospheres and the multicomponent (hyperfine) structure of the $\mathrm{Li}$ "doublet." The line absorption was then summed for each spectrum to give the synthesized equivalent width. For a program star, interpolation within the grid of observables $\left(T_{\text {eff }}, W_{\mathrm{Li}}\right)$ returns the $\mathrm{Li}$ abundance. Individual abundances are shown in Figure $6 c$. The ultra-Li-weak star G186-26 is not shown in these figures.

The random error in each abundance measurement is taken to be the quadratic sum of the components because of errors in $W$ and in the estimated temperature,

$$
\sigma_{\mathrm{err}}^{2}=\left(\frac{\partial A}{\partial \lg W}\right)^{2} \sigma_{\lg W}^{2}+\left(\frac{\partial A}{\partial T}\right)^{2} \sigma_{T}^{2} .
$$

Errors in continuum placement are incorporated in the error estimate for $W$, but errors in microturbulence, surface gravity, and metallicity are neglected entirely, being very small compared with those stated. Although we compute the error for each star individually, it is useful to make a general estimate of $\sigma_{\text {err }}$ for the ensemble using mean values from Table $2:\langle\bar{W}\rangle=21 \mathrm{~m} \AA,\left\langle\sigma_{\bar{W}}\right\rangle=1.3 \mathrm{~m} \AA$, and $\left\langle\sigma_{T}\right\rangle=$ $32 \mathrm{~K}$. Since $(\partial A) /(\partial T)=0.00065$ dex $\mathrm{K}^{-1}$ for turnoff stars, we expect $\sigma_{\text {err }} \simeq 0.033$ dex.

The standard deviation of the 22 observations is $\sigma_{\text {obs }}=$ $0.053 \mathrm{dex}$. This value is noticeably lower than the dispersion found by Thorburn (1994), who viewed the dispersion as significant, and by Spite et al. (1996) and Bonifacio \& Molaro (1997), who claimed the dispersion was within their errors. The study by Deliyannis et al. (1993) noted a range of possible dispersions depending on the composition of the sample.

The value $\sigma_{\mathrm{obs}}=0.053 \mathrm{dex}$ includes the contributions of quantifiable uncertainties in the data. We also need to consider the possibility that we have observed an admixture of stellar types not purely representative of Li plateau stars. On the second point, a striking feature of the observations is that the vast majority of the stars, 20 of the 22 measurements, fall within $0.1 \mathrm{dex}$ of the mean. The form of the distribution is shown in Figure $6 d$ as both a histogram and a stripe plot, the latter avoiding the undesirable effects of binning. These suggest a roughly Gaussian distribution about the mean with a small dispersion, plus two stars lower in abundance by $\simeq 0.14$ dex. Indeed, the standard deviation for the 20 stars within \pm 0.1 dex of the mean is a mere $\sigma_{\text {obs }}=0.036 \mathrm{dex}$. The two stars with lower abundances therefore represent $4.1 \sigma\left(\mathrm{CD}-24^{\circ} 17504\right)$ and $3.4 \sigma(\mathrm{BD}$ $+9^{\circ} 2190$ ) deviations, which we feel justified in excluding from the majority. We address the reasons they differ below, but for now we emphasize that for 20 of the 23 stars in Table 2, i.e., for $87 \%$ of the very metal-poor, halo-turnoff sample, the observed spread in Li abundance is only $\sigma_{\text {obs }}=$ 0.036 dex. Since we estimated the random error for the ensemble to be $\sigma_{\text {err }}=0.033 \mathrm{dex}$, it is clear that the vast majority of the sample is consistent with essentially zero intrinsic scatter about the mean $\sigma_{\text {int }}<0.02$ dex. In other words, the Spite Li plateau is ultrathin at the metal-poor turnoff.

Three stars are highlighted in Figures 6 and 7, where the latter shows the scatter about the Li plateau in standardized units $Z_{i}=\left[A(\mathrm{Li})_{i}-2.11\right] / \sigma_{A(\mathrm{Li}) i}$. Two of the stars were introduced above. $\mathrm{BD}+9^{\circ} 2190$ falls well below the mean but by only 2.8 times its formal error, so its position could

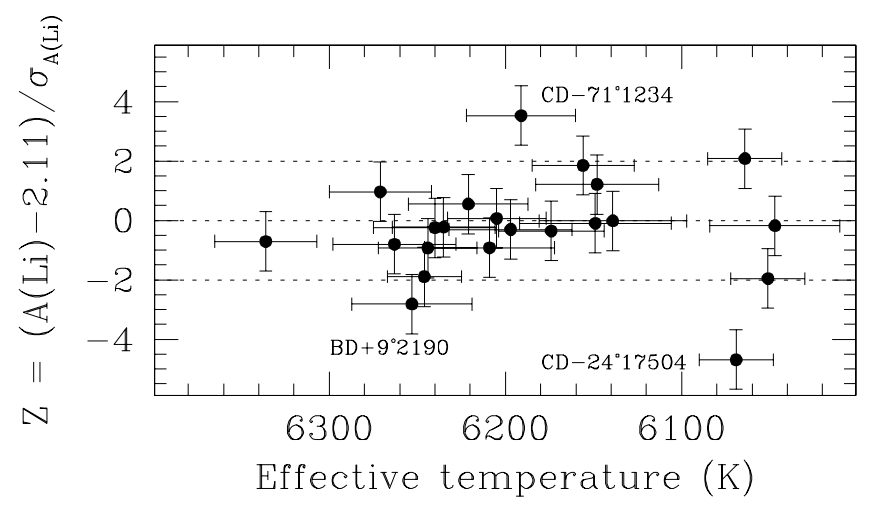

FIG. 7.- Spread in standardized residuals $\left(Z_{i}=\left[A_{i}(\mathrm{Li})-2.11\right] / \sigma_{A(\mathrm{Li}), i}\right)$ about the 2.11 dex locus. See text for discussion.

be consistent with its errors. In contrast, CD $-24^{\circ} 17504$ and $\mathrm{CD}-71^{\circ} 1234$ lie away from the mean by, respectively, 4.7 and 3.5 times their formal errors, suggesting that they have genuinely different $\mathrm{Li}$ abundances from the rest. In $\S 4.2 .3$ we searched for but failed to find evidence that the formal error estimates were unreasonable; it would be ad hoc, without more evidence, to suggest that these two stars are exceptions, especially since each has several observations. Even exclusion of $\mathrm{CD}-24^{\circ} 17504^{\prime}$ 's most extreme datum-1997: $W=15.1 \mathrm{~mA}-$ would leave the star below the mean by 3.0 times its (revised) formal error. We conclude that both stars lie significantly away from the mean and discuss the cause of this below (§ 7.3.1).

\section{DISCUSSION}

\subsection{Comparison with Previous Measurements of Spread}

The essentially zero spread found for the Li plateau at the very metal-poor turnoff may be contrasted with larger values found in several previous studies. Spite et al. (1996) and Bonifacio \& Molaro (1997) both considered the spread they measured to be consistent with zero to within their formal errors. Our new result offers no contradiction, but because of the much better precision achieved in our study, $\sigma_{\text {err }} \simeq 0.033$ dex compared with 0.06-0.08 dex (Spite et al.) and 0.07 dex (Bonifacio \& Molaro), our result can be stated much more strongly. Our better precision derives from the use of a very homogeneous data set, the checks undertaken to ensure that error estimates were appropriate (e.g., through double-blind processing and double measuring of every spectrum), the utilization of multiple indices to minimize random errors in effective temperature, and the application of restrictive selection criteria that minimized physical differences between the stars. Otherwise, star-tostar differences might have induced greater temperature and/or metallicity dependent errors associated with coloreffective temperature transformations and model atmospheres. We cannot claim that our abundance calculations have completely overcome the systematic errors-we quantify them in $\S 7.7$ - but we have avoided them insofar as they affect measurements of the thickness of the Li plateau.

Given the essentially zero intrinsic scatter found for our sample, how are we to interpret the earlier measurements of significant scatter by Deliyannis et al. (1993) and Thorburn (1994)? We reexamine these studies in reverse chronological order. 
Thorburn (1994) acquired an almost homogeneous data set, making extensive observations (utilizing four different telescope/spectrograph combinations) and quoting formal errors in the range $0.08-0.09$ dex. Although the bulk scatter in abundances was $25 \%$, this reduced to $15 \%$ once $T_{\text {eff }}$ and $[\mathrm{Fe} / \mathrm{H}]$ trends were removed. It is this latter figure that is relevant to the thickness of the Li plateau. Thorburn noted that the total formal error would have to be increased by $\sim 20 \%$ to explain the observed scatter and suggested that the scatter may be a consequence of dispersion in the halo age-metallicity relationship and Galactic chemical evolution (GCE). However, the much smaller scatter we have found, $\simeq 0.03 \mathrm{dex}$, obviates the need for such an explanation. We cannot be certain of the reason for the excess scatter in Thorburn's study, but we argue in the following that it may be artificial. Four different instrumental setups were used, but neither sky nor scattered light subtractions were made, which Thorburn estimated could introduce errors of not more than $1 \%-2 \%$ and possibly $3 \%-5 \%$, respectively. It is conceivable that differences between the scattered light and sky backgrounds from telescope to telescope and from night to night have contributed to the scatter in the data. From Thorburn's Table 2, the formal $\sigma(W)$ is typically only $10 \%$ of $W$. The errors from neglect of sky and scattered light will contribute $0 \%$ of $W$ in the optimistic case and $7 \%$ of $W$ in the pessimistic case, so the actual errors should be higher than the stated values by between 1.0 and 1.7 times. It is conceivable that the stated $\sigma(W)$ values do underestimate the actual errors in $W$ sufficiently to explain the 1.2 times higher than expected scatter.

Deliyannis et al. (1993) studied an inhomogeneous compilation of data from the literature and quantified the uncertainties of each measurement using a noise model of the type discussed above (e.g., Cayrel 1988). They considered a number of subsamples and found a dispersion of $\pm \geq 20 \%$ $(2 \sigma)$, i.e., $\sigma \geq 0.04$ dex, depending on which subsample was examined. This dispersion is not much different from our observed scatter, but as noted already, our formal errors are also at this level, so we infer less than 0.02 dex intrinsic scatter. They computed the scatter in each sample at uniform $b-y$ color, which can be viewed as removing trends in $T_{\text {eff }}$ but not trends in $[\mathrm{Fe} / \mathrm{H}]$. Claims of a dependence of $A(\mathrm{Li})$ on $[\mathrm{Fe} / \mathrm{H}]$ had not been published at the time of the Deliyannis et al. work. With the benefit of hindsight, we might expect that the Deliyannis et al. scatter measurements could be inflated by the presence of such a trend, if it exists. (We will return to that point below.) Subsequently, Thorburn (1994) estimated the $[\mathrm{Fe} / \mathrm{H}]$ dependence of the $\mathrm{A}(\mathrm{Li})$ trend as 0.13 dex per dex; Ryan et al. (1996a) derived a similar value, 0.11 . The Deliyannis et al. sample ranged from $-3.5 \leq[\mathrm{Fe} / \mathrm{H}] \leq-1.4$, which would span 0.25 dex in $A(\mathrm{Li})$ if the slope noted above were correct. A normally distributed sample has a standard deviation from $\sim \frac{1}{6}$ to $\frac{1}{4}$ of its range, so a sample spanning 0.25 dex might well be expected to yield a standard deviation of 0.04-0.06 dex. Thus the scatter derived by Deliyannis et al. is consistent with published values of the embedded metallicity dependence of $A(\mathrm{Li})$ and with the abundance range of their sample.

This explanation of Deliyannis et al.'s findings would fail, however, if the metallicity trend did not exist, as Bonifacio \& Molaro (1997) concluded. We revisit this below (§ 7.3). Bonifacio \& Molaro also pointed out that both Thorburn's and Deliyannis et al.'s work used straight line fits to the data in determining the scatter, whereas exponential fitting functions may have been more appropriate. Although theoretically a nonlinear form may have been better suited, it is not clear quantitatively whether the difference can be explained in this fashion.

\subsection{Reexamination of G64-12, G64-37, and CD - $33^{\circ} 1173$}

Ryan et al. (1996a) drew particular attention to G64-12, G64-37, and CD $-33^{\circ} 1173$ as three stars having essentially identical atmospheric parameters but irreconcilable lithium abundance determinations. All three stars are included in the present study, and as the conclusions already stated indicate, we no longer identify a significant spread among this set of stars. The effective temperatures (Table 2) are still within a total range of $30 \mathrm{~K}$, and the metallicities (Table 1) are within 0.10 dex. However, the homogeneous Li equivalent widths we have measured in this work differ considerably from those in the heterogeneous compilation of Ryan et al. The new versus old values (in $\mathrm{m} \AA$ ) are, respectively, as follows: G64-12, $21.1 \pm 1.1$ versus $27 \pm 1.8$; G64-37, $18.2 \pm 1.5$ versus $15 \pm 1.0$; and $C D-33^{\circ} 1173,17.2 \pm 1.2$ versus $12 \pm 1.2$. The formal errors in the current work differ little from the 1996 compilation, but in view of the homogeneity that we have achieved in the new data set, we prefer the newer measurements. We have no detailed explanation for the discrepancy other than to repeat the cautions given in Ryan et al. (1996a) and elsewhere that it is easy to overlook or misjudge error contributions when making error estimates and combining heterogeneous data sets.

\subsection{Examination of $A(\mathrm{Li})$ versus $[\mathrm{Fe} / \mathrm{H}]$ \\ 7.3.1. Morphology of the Sample}

Trends of $A(\mathrm{Li})$ with both $T_{\text {eff }}$ and $[\mathrm{Fe} / \mathrm{H}]$ were cited by Norris et al. (1994), Thorburn (1994), and Ryan et al. (1996a). However, Bonifacio \& Molaro (1997) concluded that these were eliminated by using the IRFM temperatures of Alonso et al. (1996b).

We chose our sample to be very metal-poor, both to minimize the differences between stars in the study and to obtain measurements of objects that show the least signs of chemical enrichment. However, the stars do span a small range of metallicity and, given the proven accuracy of the data, are useful for examining again the metallicity dependence of $A(\mathrm{Li})$. We plot $\mathrm{Li}$ abundance versus $[\mathrm{Fe} / \mathrm{H}]$ in Figure $8 a$. Recall from $\S 3$ that the $[\mathrm{Fe} / \mathrm{H}]$ values are mostly based on high- and/or medium-resolution spectroscopic observations, for which $\sigma_{[\mathrm{Fe} / \mathrm{H}]} \simeq 0.15 \mathrm{dex}$. It is clear at first glance that a similar trend with $[\mathrm{Fe} / \mathrm{H}]$ is identified in the present study as was measured by Thorburn (1994), 0.13 dex per dex, and by Ryan et al. (1996a), $0.111 \pm 0.018(1 \sigma)$. An ordinary least-squares (OLS) fit, excluding only G186-26, gives $d A(\mathrm{Li}) / d[\mathrm{Fe} / \mathrm{H}]=0.121 \pm 0.028$ (errors are standard errors), with a scatter about the trend $\sigma_{\text {obs }}=0.037$ dex (Fig. $8 a$, dotted line). Moreover, although we discussed above whether $C D-24^{\circ} 17504$ and $C D-71^{\circ} 1234$ should be included or excluded, it is clear that they lie on the same trend as the rest of the data in Figure 8a. This justifies our confidence in the quality of the observational data.

Because of possible concern whether the trend is real or illusory, we undertook a series of regression analyses, excluding a priori G186-26. These included an OLS, a reweighted least-squares (RWLS-Rousseeuw \& Leroy 1987), which is a robust technique that detects outliers, the 

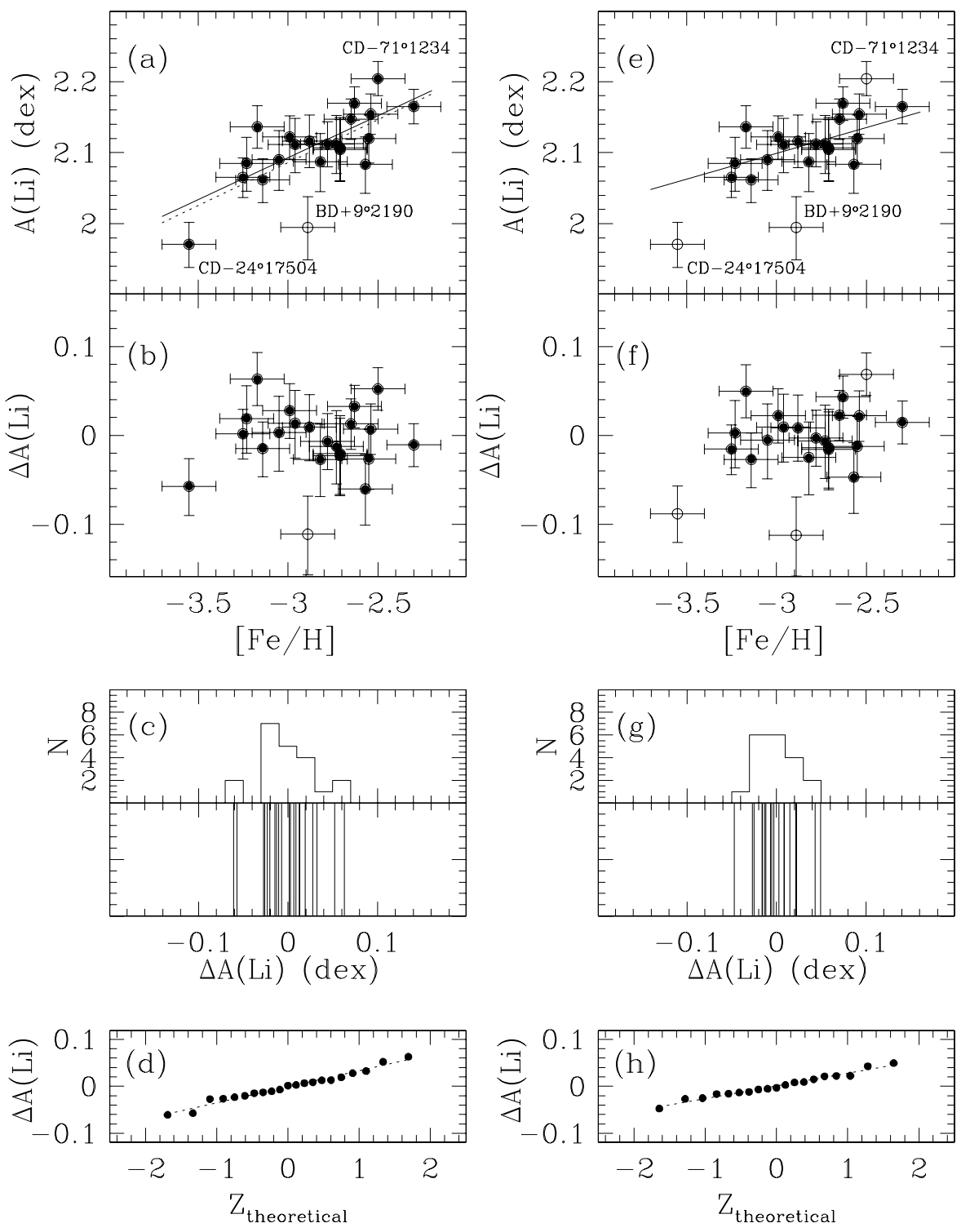

Fig. 8. - (a-d) "Best Fit" sample (G186-26 rejected a priori). (a) Dependence of $A(\mathrm{Li})$ on $[\mathrm{Fe} / \mathrm{H}]$ : univariate OLS fit for all stars (dotted line); univariate RWLS fit in which BD $+9^{\circ} 2190$ was rejected by the analysis as an outlier (solid line). This is our best-fit regression. (b) $A(\mathrm{Li})$ residuals from best fit. (c) Distribution of residuals shown as both a conventional histogram and a stripe plot. $(d)$ "Normal probability plot" confirming that the residuals are distributed normally (see text):OLS fit to guide the eye, to highlight linearity (dotted line). $(e-h)$ "Culled Fit" sample from which BD $+9^{\circ} 2190$, CD $-24^{\circ} 17504$, and $\mathrm{CD}-71^{\circ} 1234$ have been excluded, illustrating that even a culled sample yields a significant metallicity dependence. $(e)$ Dependence of $A(\mathrm{Li})$ on $[\mathrm{Fe} / \mathrm{H}]$ : univariate RWLS fit in which BD $+9^{\circ} 2190$ was excluded a priori, and subsequently $\mathrm{CD}-24^{\circ} 17504$ and $\mathrm{CD}-71^{\circ} 1234$ were rejected by the analysis as outliers (solid line). ( $f$ ) Same as for $(b) .(g)$ Same as for $(c)$. (h) Same as for $(d)$.

BCES $^{6}$ approach, which uses the errors in both quantities, and a robust technique based on a bisquare regression procedure described by $\mathrm{Li}$ (1985). The first step was to undertake OLS and RWLS bivariate analyses of $A(\mathrm{Li})=$ $a_{0}+a_{1}[\mathrm{Fe} / \mathrm{H}]+a_{2} T_{\text {eff }}$. Detailed results are presented in Table 5. The coefficient of determination, $R^{2}$, listed in the table indicates the proportion of variance in the dependent variable that is explained by the independent variable(s) in the regression model. For both techniques, and also for culled subsets of the data, we found the coefficient of $T_{\text {eff }}$ to be indistinguishable from zero, to a precision of $\simeq 0.010$ $(1 \sigma)$ dex per $100 \mathrm{~K}$. This is not entirely surprising, given the short temperature interval for the data, but it is nevertheless 1996).

${ }^{6}$ Bivariate correlated errors and intrinsic scatter (Akritas \& Bershady inconsistent $(>3 \sigma)$ with the result of a previous analysis of heterogeneous data (Ryan et al. 1996a). The bivariate RWLS analysis rejected BD $+09^{\circ} 2190$ as an outlier, but this did not alter the redundant status of the $T_{\text {eff }}$ coefficient. Whatever the explanation for the difference between the Ryan et al. (1996a) sample and the current one, clearly a temperature term is unnecessary in the present analysis, and all further tests were conducted using univariate fits of the form $A(\mathrm{Li})=a_{0}+a_{1}[\mathrm{Fe} / \mathrm{H}]$.

The OLS univariate fit to the data is shown as a dotted line in Figure $8 a$. The RWLS fit again identified BD $+9^{\circ} 2190$ as an outlier, and the fit to the remaining stars is shown with the solid line. This represents our "best fit:"

$$
A(\mathrm{Li})=[(2.447 \pm 0.066)+(0.118 \pm 0.023)][\mathrm{Fe} / \mathrm{H}] \text {. }
$$

(The RWLS regression is identical to that which would be obtained from the OLS fit if $\mathrm{BD}+9^{\circ} 2190$ was excluded a 
TABLE 5

RESUltS OF REGRESSION ANALYSES

\begin{tabular}{|c|c|c|c|c|c|}
\hline \multicolumn{2}{|c|}{ SAMPLE Size } & \multirow[b]{2}{*}{ METHOD } & \multirow[b]{2}{*}{ FIT } & \multirow[b]{2}{*}{$R^{2}$} & \multirow[b]{2}{*}{ Notes } \\
\hline Initial $^{\mathrm{a}}$ & Final & & & & \\
\hline 22 & $\ldots$ & OLS & $A(\mathrm{Li})=[(2.461 \pm 0.695)+(0.121 \pm 0.029)][\mathrm{Fe} / \mathrm{H}]+(0.000 \pm 0.011)\left(T_{\text {eff }} / 100\right)$ & 0.48 & \\
\hline 22 & $21^{\mathrm{b}}$ & RWLS & $A(\mathrm{Li})=[(2.102 \pm 0.575)+(0.118 \pm 0.024)][\mathrm{Fe} / \mathrm{H}]+(0.006 \pm 0.009)\left(T_{\text {eff }} / 100\right)$ & 0.59 & \\
\hline 22 & $21^{\mathrm{b}}$ & RWLS & $A(\mathrm{Li})=[(2.447 \pm 0.066)+(0.118 \pm 0.023)][\mathrm{Fe} / \mathrm{H}]$ & 0.58 & Fig. $8 a$, solid line \\
\hline 22 & $\ldots$ & BCES & $A(\mathrm{Li})=[(2.570 \pm 0.094)+(0.164 \pm 0.032)][\mathrm{Fe} / \mathrm{H}]$ & & \\
\hline 22 & $\ldots$ & BCES Bootstrap & $A(\mathrm{Li})=[(2.480 \pm 0.093)+(0.132 \pm 0.033)][\mathrm{Fe} / \mathrm{H}]$ & & \\
\hline 22 & $\ldots$ & Robust & $A(\mathrm{Li})=[(2.430 \pm 0.084)+(0.113 \pm 0.030)][\mathrm{Fe} / \mathrm{H}]$ & 0.48 & \\
\hline \multicolumn{6}{|c|}{ Univariate Analyses with Culled Sample, excluding BD $+9^{\circ} 2190, \mathrm{CD}-24^{\circ} 17504$, and CD $-71^{\circ} 1234$} \\
\hline 19 & $\ldots$ & Robust & $A(\mathrm{Li})=[(2.346 \pm 0.064)+(0.083 \pm 0.022)][\mathrm{Fe} / \mathrm{H}]$ & 0.39 & \\
\hline
\end{tabular}

NoTE.-Stated errors are standard errors.

${ }^{a}$ G186-26 was excluded from all analyses a priori.

${ }^{\mathrm{b}} \mathrm{BD}+9^{\circ} 2190$ was rejected by the analysis as an outlier.

${ }^{c} \mathrm{BD}+9^{\circ} 2190$ was excluded from this analysis a priori, and the other two stars were rejected by the analysis as outliers.

d This case is identical to RWLS with final sample $=19$.

priori.) Clearly, the result is barely sensitive to the inclusion or exclusion of this star. The coefficient of $[\mathrm{Fe} / \mathrm{H}]$ is found to be nonzero at a high significance, viz., $0.118 \pm 0.023(1 \sigma)$. The same conclusion was reached from the assortment of other regression tests preformed (see Table 5 for details).

Earlier in the discussion, we identified CD $-24^{\circ} 17504$ and $\mathrm{CD}-71^{\circ} 1234$ as deviating from the mean by more than their formal errors. This can now be understood in terms of their rankings at the low and high end of the metallicity scale. Although they were not identified as outliers by the RWLS fit, we considered further the possibility that they might carry excessive weight in influencing the trend and conducted tests on a culled sample. A RWLS regression from which $\mathrm{BD}+9^{\circ} 2190$ was culled a priori subsequently identified $C D-24^{\circ} 17504$ and $C D-71^{\circ} 1234$ as outliers and gave a shallower, but still significantly nonzero, slope for the trend:

$$
A(\mathrm{Li})=[(2.318 \pm 0.063)+(0.073 \pm 0.022)][\mathrm{Fe} / \mathrm{H}] .
$$

The fit for the culled sample is shown in Figure 8e. Alternative regression fits for this sample are given in Table 5; all give significantly nonzero values for the slope. ${ }^{7}$

Figures $8 b$ and $8 f$ give the residuals of $A(\mathrm{Li})$ about the regression functions, with open circles indicating data excluded from the fit. Histograms and stripe plots (Figs. $8 c$ and $8 g$ ) show the residual distributions. The best fit yields a

\footnotetext{
${ }^{7}$ We note for completeness that the star HD 74000, which fell outside the metallicity and temperature range of our sample selection criteria, was nevertheless observed as a standard star, in order that we could compare our equivalent width measurements with those of other workers. We noticed, however, that it has a lower $A(\mathrm{Li})$ abundance than most other stars in our sample. If it had been included in the target group, it too would have been rejected by the outlier-detection routines in our regression analyses. If ${ }^{7} \mathrm{Li}$ is genuinely depleted in this star, this may account for the nondetection of ${ }^{6} \mathrm{Li}$ despite it being only $100 \mathrm{~K}$ cooler than HD 84937 and $\mathrm{BD}+26^{\circ} 3578$, in which ${ }^{6} \mathrm{Li}$ is seen (Smith et al. 1998).
}

dispersion

$$
\sigma_{\text {obs }}=0.031 \mathrm{dex},
$$

while the "culled fit" has $\sigma_{\text {obs }}=0.024$ dex. Robust biweight estimators of scale (see Beers, Flynn, \& Gebhardt 1990 and references therein) yield values $S_{B I}=0.031$ and 0.025 , respectively. (The biweight estimate of scale converges to the standard deviation estimator when sampling from a normal distribution but is less sensitive to the presence of outliers). The normality of the $A(\mathrm{Li})$ residuals is established not only by the excellent agreement between the $\sigma$ and $S_{B I}$ values, but also via the lack of departure from linearity in the "normal probability plots" 8 in Figures $8 d$ and $8 h$.

The regression analysis may be thus summarized as follows: We have found a positive dependence of $A(\mathrm{Li})$ upon $[\mathrm{Fe} / \mathrm{H}]$ (but not $T_{\text {eff }}$ ), which resembles the values found previously by Thorburn (1994) and Ryan et al. (1996a). Our best fit gives $d A(\mathrm{Li}) / d[\mathrm{Fe} / \mathrm{H}]=0.118 \pm 0.023$. Shallower values of the slope can be obtained by a priori rejection of some of the data-which may be an invalid action-leading to $d A(\mathrm{Li}) / d[\mathrm{Fe} / \mathrm{H}]=0.073 \pm 0.022$, but even then the slope is significant at $\geq 3 \sigma$. The scatter measured for the best fit is $\sigma_{\text {obs }}=0.031$ dex. Obviously, rejection of stars to obtain a shallower slope yields even smaller values of the scatter, but in any case the observed scatter is consistent with the expected errors $\sigma_{\text {err }} \simeq 0.033$.

\footnotetext{
${ }^{8}$ A normal probability plot ranks the data from lowest to highest and plots the ordered value against its theoretical $Z$-statistic. The $Z$-statistic gives the number of standard deviations by which the datum would depart from the mean in a normal distribution of $N$ points. For a normal distribution, ranked datum $i$ will possess probability value $i /(N+1)$ measured from the lowest tail, so the cumulative probability distribution is inverted to find the corresponding $Z$-statistic. If a data set is normally distributed, then it will lie about a straight line in the plot, whereas an asymmetric distribution will deviate from the line along a curved path. See, e.g., Levine, Berenson, \& Stephan (1998, their § 5.6).
} 


\subsubsection{Is the Trend Natural or Artificial?}

A major similarity between the Ryan et al. (1996a) study and the present one is the use of the same computations relating equivalent width to abundance. If a metallicitydependent error existed in that work, it would persist here. Can such an error be identified?

The Ryan et al. (1996a) work used model atmospheres from R. A. Bell (1983, private communication), computed at $[\mathrm{Fe} / \mathrm{H}]=-2$. If the model structure differed sufficiently for real stars between $[\mathrm{Fe} / \mathrm{H}]=-3.0$ and -2.0 , a metallicity dependent error might be expected. However, the Kurucz (1993) models (which extend to lower abundance than Bell's but have high convective overshoot) show that changing from the higher to the lower metallicity would change the inferred Li abundance by only 0.012 dex (see Ryan et al. 1996a, their Fig. 2). This is an order of magnitude less than the trend identified and a factor of 3 smaller than $\sigma_{\text {obs }}$, in the sense of steepening rather than flattening the trend. On this estimate, the atmospheric models are not sufficiently sensitive to metallicity to produce the trend we observe. Note also that Thorburn used Kurucz (1993) models rather than the Bell models as adopted here, yet derived an almost identical trend. This emphasizes again that selection of a different model grid may alter the derived absolute abundance but will have little effect on the differential characteristics of the results.

An alternative source of error might be a metallicity dependence in the effective temperature scale. Our effective temperatures were based substantially on $B-V, b-y$, and HP2. In $\S 5$ we argued that all of the indices used are insensitive to metallicity for very low-metallicity turnoff stars. An error of $100 \mathrm{~K}$ in effective temperature would produce an abundance error of 0.065 dex for stars of the temperature and metallicity of our sample, so an error of $200 \mathrm{~K}$ would have to be induced over the short metallicity interval from $[\mathrm{Fe} / \mathrm{H}]=-2.3$ to -3.5 to produce the trend observed, yet we identified, at worst, an $18 \mathrm{~K}$ change in the Kurucz (1993) color transformations. In view of the lack of sensitivity of our temperature indicators for the types of stars investigated, we do not believe that the trend can be explained away in this fashion.

We have ruled out metallicity-dependent errors in the stellar atmospheres and effective temperature scales as causes of the trend. We do not expect such errors in the equivalent width measurements either, since the spectra are devoid of lines around $\mathrm{Li} 6707 \AA$ and the continuum fit should be reliable irrespective of metallicity for our stars. Non-LTE effects were assessed and rejected as the cause by Ryan et al. (1996a). We are left with little alternative but to restate our identification of the trend over the interval $-3.6<[\mathrm{Fe} / \mathrm{H}]<-2.3$ and to consider it to be natural until proved otherwise.

\subsubsection{The Bonifacio \& Molaro Analysis in Retrospect}

The metallicity dependence derived here is very similar to that found by Ryan et al. (1996a) and Thorburn (1994), but the new sample is far more homogeneous and of much higher quality. How then should we view Bonifacio \& Molaro's (1997) conclusion that there is no metallicity dependence? Their work used IRFM temperatures, which one might arguably prefer over other scales, especially as far as systematic errors are concerned, but there are two crucial disadvantages of their study compared with ours. First, the formal errors in the IRFM temperatures listed by Bonifacio
\& Molaro are typically $80 \mathrm{~K}$, whereas by averaging many different indices we have reduced the random error to typically $30 \mathrm{~K}$. The larger errors of the IRFM temperatures induce greater random scatter about the Li plateau for that dataset. Second, their equivalent widths and $[\mathrm{Fe} / \mathrm{H}]$ values were based on a literature survey of inhomogeneous and less reliable data than in our new work. The combined effect of these factors is that, although their bivariate fit of $A(\mathrm{Li})$ on $[\mathrm{Fe} / \mathrm{H}]$ and $T_{\text {eff }}$ gave a slope consistent with zero, the uncertainty in its determination was sufficiently large that our new value lies at only their $2.5 \sigma$ tolerance. $^{9}$ Bonifacio \& Molaro's univariate fits, however, are irreconcilable with our result, having $[\mathrm{Fe} / \mathrm{H}]$ coefficients ranging from -0.02 to -0.05 dex per dex and uncertainties $(1 \sigma)$ of $0.03-0.06$ dex per dex, depending on the statistical test. In what follows, we identify additional reasons for the differences between their result and ours obtained with the current sample.

The difference between our estimated slope of $A(\mathrm{Li})$ on $[\mathrm{Fe} / \mathrm{H}]$ and that of Bonifacio \& Molaro (1997) can be explained upon closer scrutiny of the literature data used in their study. Figure 9 shows the subset of nine stars common to both works. We use the new homogeneous $W(\mathrm{Li})$ values from this study but show abundances calculated on both the IRFM and our temperature scales (middle and upper panels, respectively). We plot these against both the literature $[\mathrm{Fe} / \mathrm{H}]$ values referenced in Table 2 (left panels) and the values used by Bonifacio \& Molaro (right panels). The effect of using the IRFM scale is to generate huge scatter (middle panels) due to the low precision of those individual values. As emphasized previously, high internal precision is required to assess the spread about the Li plateau, and this precision is delivered by the variance ${ }^{-1}$-weighted average over three to six different temperature indicators, not by the use of a single "noisy" index even if the latter may have better systematics.

Restricting our attention, then, to the low-scatter (uppermost) panels using the temperatures computed in this work, it is clear that the trend with metallicity depends on the adopted metallicity estimates. Without more information, it would not be possible to know whether the literature $[\mathrm{Fe} / \mathrm{H}]$ compilation in Table 2 or that used by Bonifacio \& Molaro (1997) is better. Fortunately, we do have more information, in the second set of $[\mathrm{Fe} / \mathrm{H}]$ values derived from applying the calibration of Beers et al. (1999) to our 1 $\AA$ resolution spectra (see Table 2). In the bottom panels of Figure 9, we compare the metallicities derived from those 1 $\AA$ resolution spectra with the adopted literature values (Fig. $9 e$ ) and those used by Bonifacio \& Molaro (Fig. 9f), and we find excellent agreement with our adopted literature values but considerable disagreement with some of the values adopted by Bonifacio \& Molaro, to the extent that the plot in Figure $9 b$ becomes leveled off by the scatter in $[\mathrm{Fe} / \mathrm{H}]$. For completeness, we note that an OLS regression of our presently derived $A(\mathrm{Li})$ estimates with the Molaro \& Bonifacio values of $[\mathrm{Fe} / \mathrm{H}]$ for the nine stars in common (Fig. $9 b$ ) results in a slope with respect to abundance of $0.008 \pm 0.041$, i.e., completely consistent with zero. However, on the basis of these comparisons, we favor the literature $[\mathrm{Fe} / \mathrm{H}]$ values adopted in Table 2 to those

\footnotetext{
9 Their bivariate fit for an LTE analysis without depletion corrections (i.e., matching our assumptions) gave a metallicity slope $0.034 \pm 0.034 \mathrm{dex}$ per dex.
} 


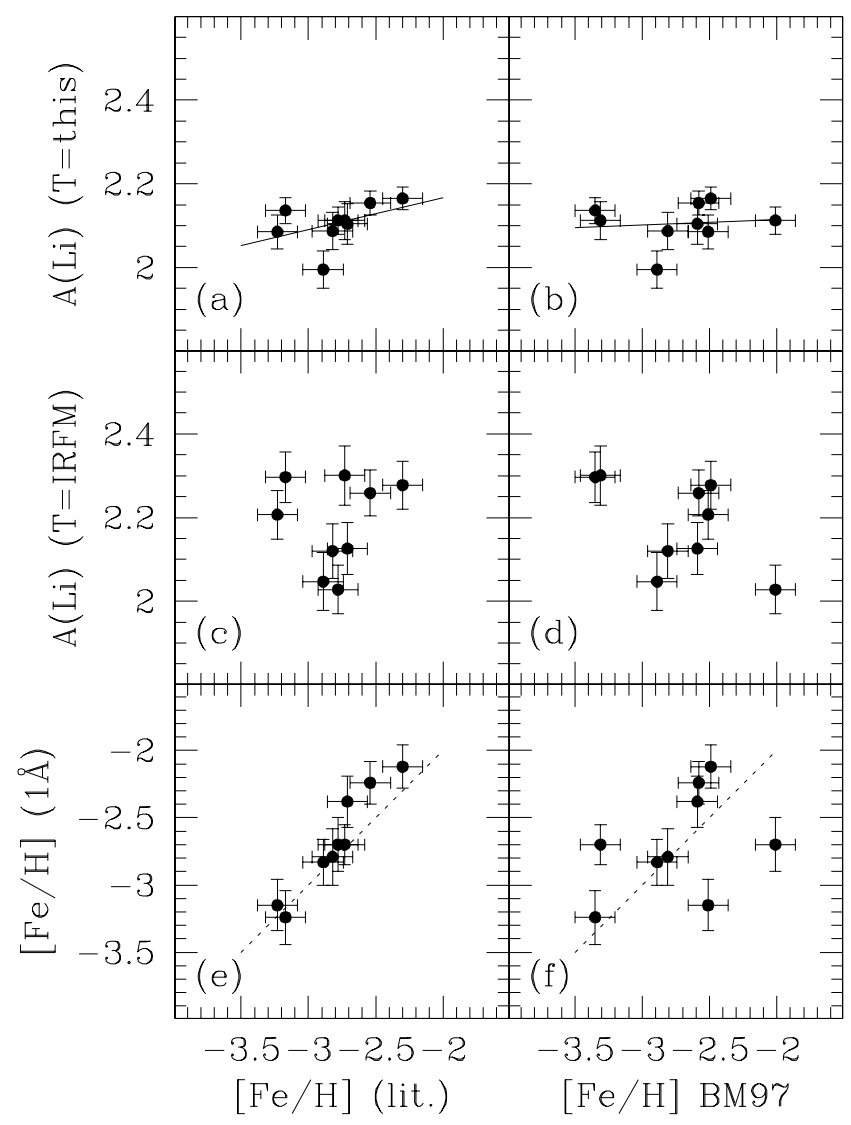

FIG. 9.- $(a-d)$ Lithium abundances derived for two sets of effective temperature values and plotted against two sets of $[\mathrm{Fe} / \mathrm{H}]$ values. The solid line shows the OLS fit. $(e-f)$ Metallicities derived from $1 \AA$ resolution spectra compare well with the high-resolution values adopted in this study but unfavorably with several values adopted by Bonifacio \& Molaro (1997). The dotted line shows the $1: 1$ locus for different $[\mathrm{Fe} / \mathrm{H}]$ scales. Because of the large scatter introduced by the IRFM temperatures and the disagreement between the $[\mathrm{Fe} / \mathrm{H}]$ values from $1 \AA$ resolution spectra and the values adopted by Bonifacio \& Molaro, we argue that $(a)$ is the most reliable presentation of the data. See text for discussion.

adopted by Bonifacio \& Molaro. Preferring also the temperatures derived from multiple indices rather than the individual temperatures based on the IRFM, we believe that Figure $9 a$ is the most reliable presentation of the data.

Restating our result above, allowing for the $[\mathrm{Fe} / \mathrm{H}]$ dependence in our sample with only G186-26 and BD $+9^{\circ} 2190$ excluded, we find a tiny dispersion, $\sigma_{\text {obs }}=0.031$ dex, for $91 \%$ of the sample. It remains now to discuss the significance of the trend with metallicity.

\section{4. ${ }^{6} \mathrm{Li}$ as a Tracer of Nonprimordial ${ }^{7} \mathrm{Li}$}

The interpretation of halo $\mathrm{Li}$ abundances would be greatly simplified if the Spite Li plateau had no dependence upon metallicity. However, we have again measured a positive dependence. Furthermore, and even if one denies the reality of this trend, the fact that at least two stars in our sample are contaminated with ${ }^{6} \mathrm{Li}$ indicates a distinctly nonprimordial origin for some of the $\mathrm{Li}$ in these stars. Smith, Lambert, \& Nissen (1993, 1998) and Hobbs \& Thorburn $(1994,1997)$ have measured the presence of ${ }^{6} \mathrm{Li}$ in HD 84937 and BD $+26^{\circ} 3578$ at the level of ${ }^{6} \mathrm{Li} /$ $\mathrm{Li}=0.06 \pm 0.03$ and $0.05 \pm 0.03$ (Smith et al. 1998), respectively. Both of these stars are in our sample. Our data are not of high enough resolving power or $\mathrm{S} / \mathrm{N}$ to measure ${ }^{6} \mathrm{Li}$ separately, but since all of the stars in our narrowly defined sample should have a similar evolutionary history and stellar structure, more likely than not they will all be contaminated by ${ }^{6} \mathrm{Li}$.

In the following discussion, we assume that the cited ${ }^{6} \mathrm{Li}$ detections are accurate, i.e., that the red wing of the $\mathrm{Li}$ doublet in those stars owes its presence to ${ }^{6} \mathrm{Li}$ and not to velocity asymmetries of the ${ }^{7} \mathrm{Li}$ feature. Tests by Smith et al. (1998) showed that the asymmetries that would be required in HD 84937 to explain the red wing of the Li doublet were well in excess of those measured on the Ca I $6162 \AA$ line. Computations of line transfer for the Sun in the presence of granulation have also failed to reveal significant errors in the results obtained for $\mathrm{Li}$ from one-dimensional models (Kiselman 1997; Uitenbroek 1998). Furthermore, we assume that all of the ${ }^{6} \mathrm{Li}$ is prestellar. Alternative possibilities were examined by Lambert (1995), who performed an initial appraisal of synthesis by Galactic cosmic rays stopped in the stellar convection zone, and by Deliyannis \& Malaney (1995), who considered synthesis by stellar flares. The former appraisal revealed potentially important production of ${ }^{6} \mathrm{Li}$ but with large uncertainties, and, on balance, Lambert viewed the mechanism as probably too inefficient. The second assessment indicated possibly significant levels of ${ }^{6} \mathrm{Li}$ production and retention in turnoff stars, but again the calculation was subject to large uncertainties associated with the (unknown) flare-history of the star.

Because ${ }^{6} \mathrm{Li}$ production at the levels measured exceeds that expected from standard Big Bang nucleosynthesis, we infer that it originates in sources associated with GCE, and we should expect GCE production to vary with $[\mathrm{Fe} / \mathrm{H}]$. Furthermore, since $\mathrm{GCE}^{7} \mathrm{Li}$ production must accompany GCE ${ }^{6} \mathrm{Li}$ production, we have to disentangle three components to the abundances we measure via a single spectral feature: primordial ${ }^{7} \mathrm{Li}, \mathrm{GCE}{ }^{7} \mathrm{Li}$, and $\mathrm{GCE}{ }^{6} \mathrm{Li}$.

Ramaty, Kozlovsky, \& Lingenfelter (1996) and Ramaty et al. (1997) give the production ratio of ${ }^{7} \mathrm{Li} /{ }^{6} \mathrm{Li}$ as $\sim 1.3-1.7$ for Galactic cosmic rays having energies and compositions consistent with Be and B synthesis. We adopt the value 1.5 in the calculations that follow. Assuming ${ }^{6} \mathrm{Li} / \mathrm{Li}=0.05$, (i.e., $\left.{ }^{6} \mathrm{Li} /{ }^{6+7} \mathrm{Li}\right)$ and making the most conservative assumption that none of the prestellar ${ }^{6} \mathrm{Li}$ has been destroyed in these turnoff stars, we would argue that $8 \%$ of the ${ }^{7} \mathrm{Li}$ and $13 \%$ of the total $\mathrm{Li}$ absorption in these stars is nonprimordial. We would therefore infer that the primordial value of ${ }^{7} \mathrm{Li}$ should be 0.06 dex lower than the observed $A(\mathrm{Li})$. If some of the ${ }^{6} \mathrm{Li}$ has been destroyed during these stars' lifetimes, as seems likely, then the GCE ${ }^{7} \mathrm{Li}$ fraction would be higher and the primordial value lower. Destruction of ${ }^{6} \mathrm{Li}$ at the turnoff is predicted to be a strong function of mass (effective temperature) and age, and standard Yale models (e.g., Deliyannis et al. 1990; Pinsonneault et al. 1992) show that depletion by 0.1 to 0.5 dex is not unreasonable and that substantially more depletion may have taken place in practice. If we assume that $50 \%$ of the prestellar ${ }^{6} \mathrm{Li}$ has been destroyed, then the GCE ${ }^{7} \mathrm{Li}$ component would be $17 \%$ of the total; $21 \%$ of the current line absorption would be due to GCE, and the primordial value would be 0.10 dex lower.

\subsection{Nonprimordial $\mathrm{Li}$ and the $[\mathrm{Fe} / \mathrm{H}]$ Dependence}

Since the presence of ${ }^{6} \mathrm{Li}$ indicates that at least some nonprimordial $\mathrm{Li}$ is present, it is logical to ask whether the inferred GCE ${ }^{6} \mathrm{Li}$ and ${ }^{7} \mathrm{Li}$ components can explain the observed dependence of $A(\mathrm{Li})$ on $[\mathrm{Fe} / \mathrm{H}]$. The calculations 
above show that the primordial ${ }^{7} \mathrm{Li}$ abundance probably is at least 0.06 dex lower than the $A(\mathrm{Li})$ abundance measured in HD 84937 and $\mathrm{BD}+26^{\circ} 3568$, and that a value $0.10 \mathrm{dex}$ lower might be a realistic estimate. According to the measured trend, the $[\mathrm{Fe} / \mathrm{H}]$ value at which $A(\mathrm{Li})$ is observed to be 0.10 dex lower than in the relatively metal-rich stars HD 84937 and $\mathrm{BD}+26^{\circ} 3568$ is $[\mathrm{Fe} / \mathrm{H}]=-3.2$. It is important to note that we have argued elsewhere (e.g., Ryan, Norris, \& Bessell 1991; Ryan et al. 1996b; Ryan 1996), in work not involving $\mathrm{Li}$, that the Galaxy's first supernova enrichment events give rise to stars from around $[\mathrm{Fe} / \mathrm{H}] \sim-4.0$ to -3.5 . The metallicity dependence we have measured for $\mathrm{Li}$ is therefore roughly consistent with the GCE contribution to Li inferred up to the time when HD 84937 and BD $+26^{\circ} 3568$ formed. It is not unreasonable to suppose, then, that the most metal-poor star in our sample, CD $-24^{\circ} 17504$ with $[\mathrm{Fe} / \mathrm{H}]=-3.55$, has minimal GCE contribution to its $\mathrm{Li}$ line, whereas at higher metallicities we see the GCE contribution increasing.

If ${ }^{6} \mathrm{Li}$ preservation is confined to turnoff stars, as the Yale models suggest (Deliyannis et al. 1990; Pinsonneault et al. 1992), then in cooler dwarfs we would have to adjust only for GCE ${ }^{7} \mathrm{Li}$ to obtain the uncontaminated primordial value. However, since GCE ${ }^{6} \mathrm{Li}$ contributes less than GCE ${ }^{7} \mathrm{Li}$ to the contamination, the offset would be reduced only from 0.10 dex to 0.08 dex. This is not a major difference, but it does emphasize that the metallicity dependence may be slightly weaker in dwarfs away from the turnoff.

We have argued that the observed metallicity dependence in this very metal-poor sample is consistent with the GCE contribution inferred from the ${ }^{6} \mathrm{Li}$ measurements in two stars at $[\mathrm{Fe} / \mathrm{H}] \sim-2.4$. However, if we are to claim to understand the slope as due to GCE, and hence be able to infer that the most metal-poor stars yield the correct primordial $\mathrm{Li}$ abundance, then we also need to assess whether the explanation correctly predicts the metallicity dependence in more metal-rich stars. Smith et al. (1998), among others, have noted that if the $\mathrm{Li} / \mathrm{Be}$ ratio is maintained in GCE production throughout formation of the halo, then the Galaxy ought to have become very rich in $\mathrm{Li}$ by $[\mathrm{Fe} /$ $\mathrm{H}]=-1$, but apparently it did not. The $\alpha+\alpha$ fusion mechanism produces roughly uniform $\mathrm{Li}$ throughout the phase of halo formation, in contrast to the strong metallicity dependence of Be and B (Steigman \& Walker 1992), and Olive \& Schramm (1992, their eq. [6]) predict, by comparing $\mathrm{Li}$ with $\mathrm{Be}$, a very shallow relationship, approximately $\mathrm{GCE} A\left({ }^{7} \mathrm{Li}\right) \simeq 1.59+2 Z / Z_{\odot}$ for $Z / Z_{\odot}<0.1$. Clearly, however, the detailed evolution of ${ }^{6} \mathrm{Li}$, and therefore of GCE ${ }^{7} \mathrm{Li}$, also depends on the chemical evolution model adopted (e.g., Prantzos, Casse, \& Vangioni-Flam 1993; also contrast Figs. 1 and 2 of Yoshii, Kajino, \& Ryan 1997). The lesson from these models is that the inferred total Li abundance need not climb significantly more steeply over the range $-2.5<[\mathrm{Fe} / \mathrm{H}]<-1.5$ than it does over the interval $-3.5<[\mathrm{Fe} / \mathrm{H}]<-2.5$, which we have measured. Furthermore, since the higher metallicity samples often include cooler stars, the average observed slope may flatten slightly at higher metallicity because of the erasure of the ${ }^{6} \mathrm{Li}$ contribution to $A(\mathrm{Li})$.

In summary, we regard the slope in $A(\mathrm{Li})$ versus $[\mathrm{Fe} / \mathrm{H}]$ to be concordant with the amount of GCE inferred from the observed ${ }^{6} \mathrm{Li}$ abundances. Furthermore, GCE models that have higher Li/Be yields at lower metallicity (e.g., Steigman \& Walker 1992) suggest that the amount of GCE Li expected at higher metallicities need not invalidate this explanation. Irrespective of whether the metallicity trend is believed (since there may be skeptics in the readership), from the observed ${ }^{6} \mathrm{Li}$ fractions we infer that the primordial abundance is $\simeq 0.10$ dex below that with which stars having $[\mathrm{Fe} / \mathrm{H}] \sim-2.4$ were born.

In addition to the Galactic cosmic-ray mechanism discussed above, stellar nucleosynthesis of ${ }^{7} \mathrm{Li}$ may contribute to the measured trend. D'Antona \& Matteucci (1991) computed an increase of $A(\mathrm{Li})$ by 0.17 dex over the interval from $[\mathrm{Fe} / \mathrm{H}]=-2.5$ to -1.5 for production in $2-8 M_{\odot} \mathrm{AGB}$ stars. Although that slope is subject to uncertainties in the adopted parameters - they also computed models which showed steeper trends - and could be less, it emphasizes that even at this early stage of GCE, we must recall the likelihood, not merely the possibility, that the Li we observe in halo stars is affected by GCE. The trend we have measured, 0.12 dex per dex, is concordant with the observed ${ }^{6} \mathrm{Li}$ contamination and expected stellar production.

Before leaving this discussion, we note that the narrowness of the $\mathrm{Li}$ spread is maintained over the range $[\mathrm{Fe} / \mathrm{H}]<-2.3$, even though $\mathrm{GCE}$ is leaving its mark on material, increasing $A(\mathrm{Li})$ and producing measurable ${ }^{6} \mathrm{Li}$. This result sets an additional constraint on GCE models of lithium processing. One interpretation is that any age spread in the formation of halo stars over this lowmetallicity interval must not be so great as to lead to expectations of a measurable range of $A(\mathrm{Li})$ at a given $[\mathrm{Fe} / \mathrm{H}]$. However, an inference on age ranges may be relaxed in the Searle \& Zinn (1978) framework, where early halo star formation began in separate, independently evolving fragments. In these first star formation events in the voluminous protohalo, it is possible that regions were sufficiently separated that cosmic rays accelerated in one part did not reach and induce reactions in the others, so Li would evolve in concert with the local metallicity rather than the Galactic age. Unfortunately, models of cosmic-ray propagation in the voluminous protohalo are less well constrained than in the Galactic disk, for which we can infer present day lifetimes, path lengths, and spectra. The measurement of ${ }^{6} \mathrm{Li}$ in more halo stars will help constrain the Galactic cosmic-ray production ratios ${ }^{6,7} \mathrm{Li} / \mathrm{Be}$ and ${ }^{6,7} \mathrm{Li} / \mathrm{B}$ in the earliest phase of GCE.

\subsection{Constraints on Rotationally Induced Mixing Models}

The rotationally induced turbulent mixing models of Pinsonneault et al. (1992) differ from the Yale "standard" and "diffusive" models in predicting substantial ( $\sim 1$ dex) depletion of Li in halo turnoff stars. Among the signatures of this depletion mechanism are a mildly arched "plateau" and a spread in final abundances reflecting the range of initial angular momenta of the stars.

Using updated models having an improved treatment of the evolution of angular momentum, and considering the spread in $A(\mathrm{Li})$ seen in Thorburn's (1994) data, Pinsonneault et al. (1998) concluded that the mean Spite Li plateau abundance was depleted by $0.2-0.4$ dex from the primordial value. Utilizing our more accurate data for turnoff stars, we now revisit that result.

Pinsonneault et al. (1998) computed the depletion $-\log D_{7}=A(\mathrm{Li})_{\text {final }}-A(\mathrm{Li})_{\text {initial }}$ for stars with $[\mathrm{Fe} /$ $\mathrm{H}]=-2.3$ and $T_{\text {eff }}=6000 \mathrm{~K}$, coincidentally very similar to the parameters typical of our sample. They present their results for three different solar angular momentum histories 
(which affect the calibration of their models), convolved with observational errors of 0.00 and $0.09 \mathrm{dex}$, corresponding to perfect observations and the formal error of Thorburn (1994), respectively. Because our formal errors are only 0.033 dex, we estimated the broadening required for Pinsonneault et al.'s "perfect," minimal depletion "s0" model and compare it with our data in Figure 10a. (G186-26 is heavily depleted and lies off-scale.) The depletion curves and data are brought into coincidence by assuming an initial abundance $A(\mathrm{Li})_{i}=2.22$ dex. It appears at first sight that the data are clustered more tightly than the theoretical boundaries enclosing $\pm 47.5 \%$ of the population (dashed
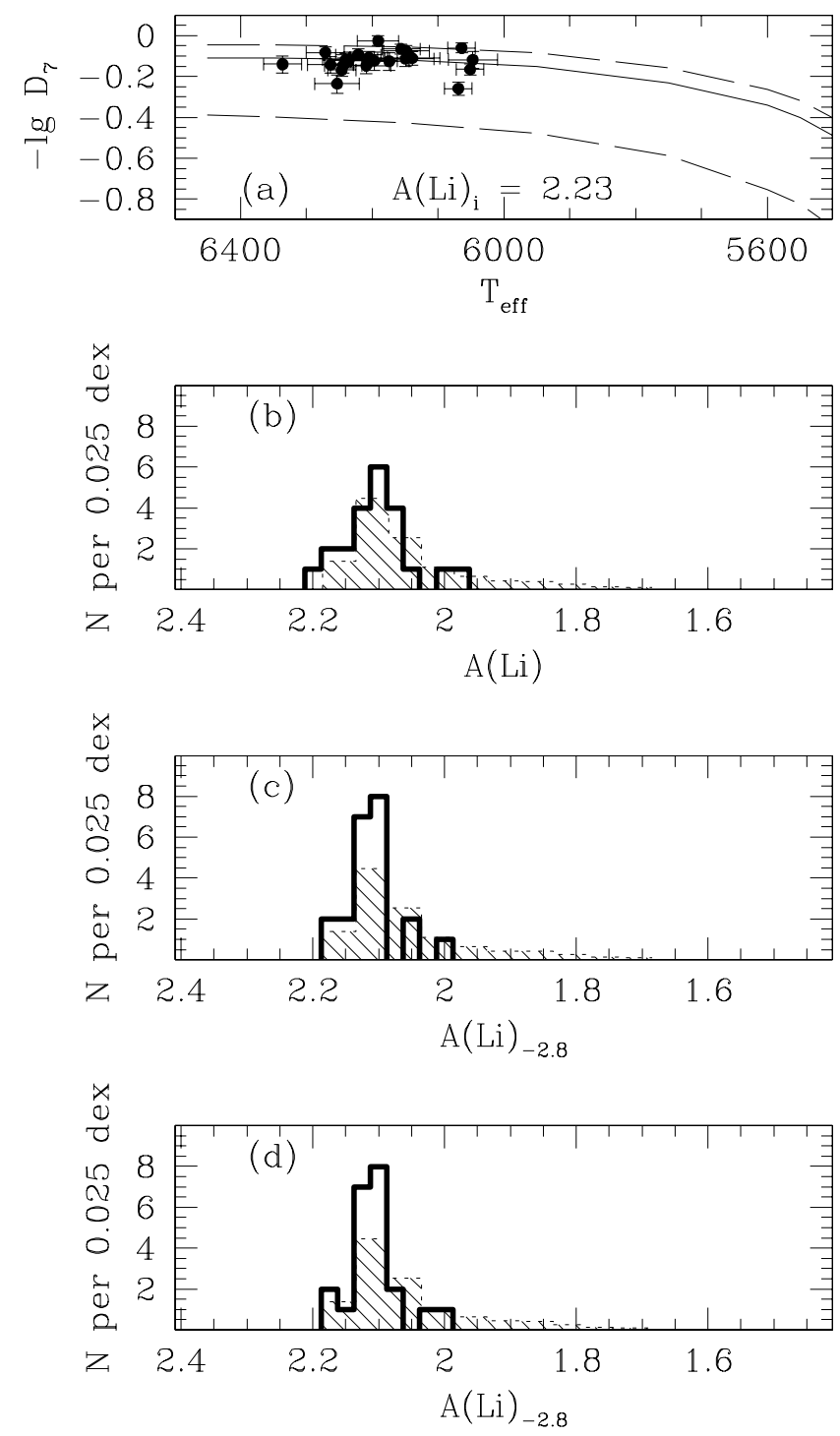

FIG. 10.-(a) Depletion curves from Pinsonneault et al. (1998) "s0" model, broadened for formal errors of 0.033 dex: median depletion (solid curve) and boundaries enclosing $\pm 47.5 \%$ of the population (dashed curves). The observational data have been superposed for an assumed initial $A(\mathrm{Li})_{i}=2.23$. (b) s0 model renormalized to our sample size and broadened for errors of $0.03 \mathrm{dex}$, assuming $A(\mathrm{Li})_{i}=2.23$ (shaded histogram); observations uncorrected for embedded metallicity dependence (heavy histogram). (c) s0 model same as for (b) (shaded histogram); observations offset to $[\mathrm{Fe} / \mathrm{H}]=-2.8$ according to the best fit to compensate for the embedded metallicity dependence (heavy histogram). The model predicts a Li-depleted tail comprising $17 \%$ of the sample, but it is not populated by the observations. (d) Same as for (c), but using the shallower slope derived from the culled sample (shaded histogram). The Li-depleted tail predicted by the model still is not populated. curves). However, it is more reliable to view the distributions functions directly, so we have renormalized the theoretical distribution with zero observational error (Pinsonneault et al.; their Fig. 9a) to the number of stars in our sample, scaled the depletion from their $T_{\text {eff }}=6000 \mathrm{~K}$ to the mean of our sample, $T_{\text {eff }}=6200 \mathrm{~K}$, and applied a broadening function to approximate the formal errors in our work. Our raw sample (excluding only G186-26), shown in Figure 10b, not surprisingly has a broader core than the theoretical distribution because of the imbedded $[\mathrm{Fe} / \mathrm{H}]$ trend. Figure $10 c$ detrends the data by shifting all stars to a common metallicity: $[\mathrm{Fe} / \mathrm{H}]=-2.8$, the median value of our sample, using $\Delta A(\mathrm{Li}) / \Delta[\mathrm{Fe} / \mathrm{H}]=0.12$. The model has a Li-depleted tail extending to much lower abundances than the data. Specifically, Figure $10 c$ for Pinsonneault et al.'s (1998) model s0 (with least depletion) predicts that $17 \%$ of the sample, or 3.9 stars for our sample of 23 , will have $A(\mathrm{Li})<2.0($ at $[\mathrm{Fe} / \mathrm{H}]=-2.8)$. In fact, we observe only one star below this limit, G186-26, which is excessively depleted compared with the model, as if some factor other than the Pinsonneault et al. mechanism is responsible. In a Poisson distribution with expected value 3.9, the probability of finding $\leq 1$ object is 0.10 ; i.e., there is only a $10 \%$ chance that the lack of objects observed in the tail is consistent with the model. Equivalently, there is $90 \%$ probability that they are inconsistent. Whereas this Poisson test examines just the tail of the distribution, the KolmogorovSmirnov test (e.g., Siegel \& Castellan 1988) compares the two distributions in their entirety. The latter (one-tail, twosample test) suggests a less than $2 \%$ probability that the tail is underpopulated only by chance; i.e., there is greater than $98 \%$ probability that the two distributions are intrinsically different. The Wilcoxon-Mann-Whitney test leads to a similar result. Figure 10d, which uses the shallower slope from the culled sample to correct for the underlying trend, still does not populate the tail, and the statistical tests give similar results.

We conclude that even the minimally depleting s 0 model of Pinsonneault et al. (1998) overpredicts the degree of $\mathrm{Li}$ depletion in the turnoff stars. Whereas the Thorburn sample allowed Pinsonneault et al. to infer depletion by $0.2-0.4$ dex by this mechanism, the higher quality data now available give rise to two new conclusions: (1) even the s0 rotational model with a median depletion as small as $0.1 \mathrm{dex}$ at $T_{\text {eff }}=$ $6200 \mathrm{~K}$ predicts a broader spread than permitted by the turnoff observations, and (2) the very low Li abundance in G186-26 is not consistent with the rotational-depletion distribution function. The latter result signals that this star, and consequently the other ultra-Li-depleted halo dwarfs, do not represent the tail of a rotational depletion distribution. It is no longer possible to infer a minimal rotational depletion of 0.2 dex as Pinsonneault et al. were led to do with less accurate data.

The extremely tight clustering of the halo turnoff stars therefore presents a serious challenge to inferences from this class of models that the turnoff stars have depleted by even as little as 0.1 dex from a higher initial value.

\subsection{The Primordial ${ }^{7} \mathrm{Li}$ Abundance}

Several estimates of the primordial Lithium abundance, $A(\mathrm{Li})_{p}$, can be made from the discussion above. They are as follows: (1) $A(\mathrm{Li})_{p}$ is $\simeq 0.10$ dex below that observed in $\mathrm{HD}$ 84937 and $\mathrm{BD}+26^{\circ} 3578$, using the ${ }^{6} \mathrm{Li}$ observations and depletion estimates to infer the underlying primordial ${ }^{7} \mathrm{Li}$ 
value; (2) it is the value measured in the most metal-poor star of the sample, $\mathrm{CD}-24^{\circ} 17504$ at $[\mathrm{Fe} / \mathrm{H}]=-3.55$, whose metals reveal a minimally processed sample of early Galactic material; or (3) it is the extrapolation of the metallicity trend to $[\mathrm{Fe} / \mathrm{H}]=-4$ where the most metal-poor stars known are found and where the metallicity distribution of the halo shows signs of truncation (Beers et al. 1998; Norris 1999). The values obtained are (1) $A(\mathrm{Li})_{p}=2.06$, (2) 1.97, and (3) 1.98. That is, we infer that the primordial abundance is $A(\mathrm{Li})_{p} \simeq 2.00$ and that future measurements of stars with $[\mathrm{Fe} / \mathrm{H}]<-3.0$ will yield values of $A(\mathrm{Li})$ lower than the bulk of the present sample [for which $A(\mathrm{Li}) \simeq 2.1$, concordant with the trend shown in Figure $8 a$. What uncertainties should we attach to our estimate of the primordial value? We refer readers to the comprehensive discussion of errors by Thorburn (1994, that paper's $\S 5$ ) and to our previous works (Norris et al. 1994; Ryan et al. 1996a), and we summarize below the results most relevant to the present discussion.

We have previously noted that typical random errors in our estimation of $A(\mathrm{Li})$ are $\sigma_{\text {err }}=0.033$ dex. Among systematic errors, Thorburn gives the uncertainties in oscillator strengths as $\sigma \simeq 0.02$ dex. A 0.5 dex error in $\lg g$ would produce less than 0.01 dex error in $A(\mathrm{Li})$ at the turnoff. Reasonable uncertainties in microturbulence and the damping coefficient are similarly unimportant because of the dominance of thermal broadening in the core of this weak line of a species with such low atomic mass. Corrections for non-LTE are -0.01 at $6100 \mathrm{~K}$ and $-0.03 \mathrm{dex}$ at $6300 \mathrm{~K}$ (for $[\mathrm{Fe} / \mathrm{H}]=-2$ and $\lg g=4$; Carlsson et al. 1994). Far greater systematic uncertainties arise as a result of the uncertainties in the zero point of the effective temperature scale and the model structures. In $\S 5.3$, we found it necessary to make zero-point adjustments to the various scales by as much as $165 \mathrm{~K}$, which for the turnoff stars corresponds to an $A(\mathrm{Li})$ change of 0.11 dex. Adoption of a different zero point would not change the spread in the plateau but would alter the inferred primordial abundance by +0.065 dex for a $100 \mathrm{~K}$ increase in the stellar $T_{\text {eff }}$ values. A similar difference arises in the abundances derived from the Bell models compared with those from Kurucz's (1993) convective overshoot models, the latter giving $A(\mathrm{Li})$ higher by 0.08 dex at the turnoff (Ryan et al. 1996a, that paper's $\S 3.3)$. Bonifacio \& Molaro's (1998) study of the Li $6140 \AA$ line in HD 140283 shows that abundances derived from the $6707 \AA$ resonance doublet are not grossly in error.

Since $\sigma_{\text {obs }}=0.031 \mathrm{dex}$ and $\sigma_{\text {err }}=0.033 \mathrm{dex}$, we have established that there is no intrinsic spread about the $\mathrm{Li}$ plateau at the metal-poor turnoff. It is clear that the absolute uncertainties in the primordial abundance are dominated not by random errors but by four systematic factors: (1) the zero point in the metal-poor effective temperature scales, $\simeq 0.1 \mathrm{dex} ;(2)$ uncertainties in the metal-poor model atmosphere structures, $\simeq 0.1 \mathrm{dex}$; (3) correction of the observed level for the contamination of $\mathrm{GCE}{ }^{6} \mathrm{Li}$ and $\mathrm{GCE}$ ${ }^{7} \mathrm{Li}$; and (4) correction for any destruction of prestellar $\mathrm{Li}$. Our three approaches (above) to account for the GCE fraction gave results ranging over 0.09 dex. That is, sources 1,2 , and 3 each contributes $\simeq 0.1$ dex to the systematic uncertainty in $A(\mathrm{Li})_{p} \simeq 2.00$.

Until recently, source 4 was perhaps the most uncertain, since the degree of depletion predicted by models depends very much on the input physics. The simplest models predict essentially no destruction of $\mathrm{Li}(<0.05 \mathrm{dex})$ at the metal-poor turnoff (Deliyannis et al. 1990). Observations show that diffusion has not affected $A(\mathrm{Li})$ (Ryan et al. 1996a), since they do not show the downward curvature of the plateau at the highest temperatures, although it is unclear how diffusion is inhibited. Vauclair \& Charbonnel (1995) suggest that small stellar winds balance diffusive effects while avoiding nuclear burning. Rotationally induced mixing led Pinsonneault et al. (1998) to infer destruction by $0.2-0.4$ dex. However, the observations presented in this work set much tighter constraints on the degree of rotationally induced mixing than the data available to Pinsonneault et al. could do, and on the basis of the very narrow scatter we have measured, we conclude that depletion by the rotationally induced mixing mechanism is less than 0.1 dex. Although this limit is more severe than Pinsonneault et al. were able to establish, it is consistent with Fields \& Olive's (1999) limit of less than 0.2 dex depletion of ${ }^{7} \mathrm{Li}$, argued on the basis of light isotope ratios.

Although the simplest models present an incomplete picture and fail to explain many behaviors (e.g., Deliyannis 1995), they may yet be giving the correct result for the turnoff stars. Certainly the thinness of the Li plateau argues against the models with rotationally induced mixing, for which a larger spread in $A(\mathrm{Li})$ is predicted. Economy of hypothesis in this situation suggests that systematic error source 4 is rather small. However, Vauclair (1999) challenges empirical inferences of this sort in the face of current models in which depletion seems unavoidable. Another possibility requiring further study is discussed in $\S 7.8$.

We finish this section by noting that the essentially zero scatter found for the very metal-poor turnoff stars points strongly toward there having been a primordial value for ${ }^{7} \mathrm{Li}$, and near-elimination of the concerns over its depletion in these stars (but see Vauclair 1999 for an opposite view) suggests that we are now closer to identifying that value with confidence. Burbidge \& Hoyle (1998) have considered that of the three factors, (1) stellar processing, (2) Galactic production, and (3) Big Bang nucleosynthesis, factor 3 is the one that has not operated. The results of the current study drive us to the contrary conclusion that factor 1 has not operated significantly, that factor 2 can be constrained jointly by the ${ }^{6} \mathrm{Li}$ abundance of these objects and the measured dependence of $A(\mathrm{Li})$ on $[\mathrm{Fe} / \mathrm{H}]$, and that factor 3 is the most likely cause for the near-uniformity first reported by Spite \& Spite (1982), supporting their conclusion that the observed abundance was "hardly altered" from the primordial one.

\subsection{The Spread in Lithium Abundances in Globular Clusters}

A discussion of the spread of $\mathrm{Li}$ in field stars would not be complete without reference to the observations of $\mathrm{Li}$ in subgiants in the globular cluster M92 that show a range of A(Li) (Deliyannis et al. 1995; Boesgaard et al. 1998). Those authors considered whether various $\mathrm{Li}$ production mechanisms - the neutrino process in SN II, Galactic cosmic-ray $\alpha+\alpha$ nucleosynthesis, and ${ }^{7} \mathrm{Be}$ transport in AGB stars - could account for the diversity, but in each case they found requirements that violated other observational constraints, such as expectations of enhanced $[\mathrm{Mg} / \mathrm{Fe}]$ ratios, age spreads within the cluster itself, and enhanced abundances of $s$-process elements. They were driven to prefer scenarios in which the range of $A(\mathrm{Li})$ reflected differential depletion from a higher abundance, rather than differential enhancement from a lower level. 
It is perhaps surprising that M92 reveals a spread in abundance of a factor of 2-3 for a small sample of stars, whereas in the field we find no spread $\left(\sigma_{\text {int }}<0.02 \mathrm{dex}\right)$ for $91 \%$ of our sample. The mean metallicities of our samples are not greatly dissimilar, and the stellar masses must be almost identical since our sample is right at the turnoff and the M92 sample is on the subgiant branch. Moreover, the globular cluster sample should have an even narrower age distribution than the field sample. Either some feature of the globular cluster environment or the different post-mainsequence evolution of the subgiants must be responsible for the differences, assuming both data sets are reliable.

Can we reconcile Boesgaard et al.'s preference for a rotationally induced depletion mechanism in the globular clusters with the absence of a spread in the halo? Possibly. If environmental factors are responsible for the difference, we may question whether the globular cluster members experience a very different history of angular momentum evolution, giving rise to a larger spread in $A(\mathrm{Li})$. Certainly the suggestion of different angular momentum distributions between cluster and field star samples is not new. Peterson, Tarbell, \& Carney (1983) and Peterson (1983) first demonstrated that the projected rotational velocities of horizontal branch stars in globular clusters, having values of $v \sin i$ up to $30 \mathrm{~km} \mathrm{~s}^{-1}$, are significantly higher than in their field counterparts, and speculation has long existed that the ubiquitous chemical abundance anomalies in globular clusters, which in many cases appear to have a bimodal signature and which are absent among halo field stars, are also driven by different angular momentum profiles (Norris 1981; Suntzeff 1981). While no satisfactory model currently exists to explain the rich and somewhat bewildering literature on globular cluster abundance variations (now known to involve C, N, O, Mg, Na, Al, Ba, and $\mathrm{Eu}$ (see Sneden et al. 1997 and references therein ${ }^{10}$ ), the signatures of abundance variations have been found even at or near the mainsequence turnoffs of some clusters (e.g., 47 Tuc (Briley et al. 1996), NGC 6752 (Suntzeff \& Smith 1991), and, most importantly in this context, M92 (King et al. 1998). In M92, King et al. report ranges in the abundances of $\mathrm{Mg}, \mathrm{Na}$, and $\mathrm{Ba}$ in the same stars for which $\mathrm{Li}$ variations have been found, although they were unable to discern any systematic correlation between the behavior of $\mathrm{Li}$, on the one hand, and the heavier elements, on the other.

While most efforts to understand the abundance anomalies have centered on the angular momentum distribution within individual stars, under the supposition that internal rotation might drive mixing, this provides an inadequate explanation for the existence of variations at and below the main-sequence turnoff ( $\mathrm{Da}$ Costa $\&$ Demarque 1982). Alternatively, one might speculate on pre-main-sequence origins for the phenomenon, and interactions between crowded protostellar disks have been proposed by R. P. Kraft (1998, private communication) as a possible mechanism for generating different abundance patterns in cluster environments. In this context, then, is it possible that interactions between the disks in the dense cluster environment enforce a diversity of evolutionary paths for the stars' angular momenta, which then affect the Li profiles in these objects? Because most of the Li depletion and dispersion in the rotationally

\footnotetext{
${ }^{10}$ For simplicity, we exclude from discussion the even more complicated abundance patterns of the cluster $\omega$ Centauri (Norris \& Da Costa 1995).
}

induced turbulent models occurs before $0.3 \mathrm{Gyr}$ (Pinsonneault et al. 1992, their Fig. 7), it is possible that the crowded cluster environments are affected by interactions between protostellar disks at just this crucial, early phase, producing different initial conditions to those found in lower density star clusters that ultimately dissolved to form the field population. If the environmental conditions have given rise to different Li-processing histories and generated different $A(\mathrm{Li})$ spreads, then we must ask again whether the thinness of the field Li plateau signifies a lack of depletion or merely depletion under conditions that were similar from one field star to another.

Alternatively, the $A(\mathrm{Li})$ spread in $\mathrm{M} 92$ may be due to some other unidentified cause, which may possibly also explain the high abundance in the field star BD $+23^{\circ} 3912$ (King, Deliyannis, \& Boesgaard 1996). In the field population, such enigmatic stars appear to be even less common than the ultra-Li-depleted stars, so it may be appropriate to regard them (or "it") as rare pathological cases not requiring us to lose sight of the "health" of the majority of Li plateau stars. Purists may argue, with some merit, that the Population II lithium origin cannot be determined with certainty until all such exceptions are understood. The observations in M92 raise the interesting possibility that globular cluster stars may exhibit quite different $\mathrm{Li}$ processing histories than the field stars. We stand to learn more not only about Li but also about the differences in globular cluster and low-density cluster environments from more detailed study, at higher $\mathrm{S} / \mathrm{N}$, of additional stars in this and other globular clusters.

\section{CONCLUDING REMARKS}

We have again found a strong dependence of $A(\mathrm{Li})$ on metallicity,

$$
d A(\mathrm{Li}) / d[\mathrm{Fe} / \mathrm{H}]=0.118 \pm 0.023 \text { dex per dex },
$$

which is concordant with theoretical GCE models and with observed ${ }^{6} \mathrm{Li}$ levels. G186-26, being ultra-Li-depleted, was rejected (ab initio) from the analysis; it is a reminder that some stars deplete their Li by 1 dex or more. The vast majority $(91 \%)$ of our very metal-poor, main-sequence turnoff, field sample is consistent with an observed scatter of only

$$
\sigma_{\text {obs }}=0.031 \mathrm{dex}
$$

about the metallicity trend. $\mathrm{BD}+9^{\circ} 2190$ was rejected by the outlier-detection algorithm from the "best" sample on account of an anomalous abundance compared with the other stars. Even so, the larger formal errors associated with this star make it unclear whether it is genuinely depleted or merely an inferior observation. Its inclusion in the best sample would have inflated the observed scatter to only $0.037 \mathrm{dex}$, compared with the expected errors $\sigma_{\text {err }}=0.033$ dex, so irrespective of its status we conclude that the intrinsic scatter of $A(\mathrm{Li})$ for the metal-poor turnoff is

$$
\sigma_{\text {int }}<0.02 \text { dex . }
$$

Several systematic uncertainties are discussed. Three involve the adopted temperature scale, the atmospheric models, and interpretation of the GCE contamination revealed by ${ }^{6} \mathrm{Li}$ and the metallicity trend. The systematic uncertainty is $\simeq 0.10 \mathrm{dex}$ in each of these. A fourth systematic uncertainty surrounds possible depletion of the pres- 
tellar $\mathrm{Li}$. The inferred intrinsic scatter is essentially zero, $\sigma_{\text {int }}<0.02$. This is much less than the range expected for the rotationally induced turbulent mixing mechanism of Pinsonneault et al. (1998), and we conclude that depletion by that mechanism must be less than $0.1 \mathrm{dex}$. The only substantial correction required to the observed abundance is for GCE, leading to a primordial abundance lower than the plateau mean. We infer $A(\mathrm{Li})_{p} \simeq 2.00 \mathrm{dex}$. This rests on the effective-temperature zero-point set by Magain's (1987) and Bell \& Oke's (1986) $b-y$ calibrations of metal-poor stars and the model atmospheres from R. A. Bell (1983, private communication), which do not possess the convective overshoot used in Kurucz's (1993) models. Use of a different effective temperature scale would increase the inferred primordial abundance by $0.065 \mathrm{dex}$ for a $100 \mathrm{~K}$ increase in the adopted $T_{\text {eff }}$ values.

The difference between our field star observations and the M92 data of Boesgaard et al. (1998) suggests that real fieldto-cluster differences in $\mathrm{Li}$ evolution may have occurred. These may indicate different angular momentum evolutionary histories, possibly associated with interactions between protostellar disks in the dense globular cluster environments. Further accurate study of Li in globular clusters will be required.

The authors gratefully acknowledge discussions with C. P. Deliyannis and J. A. Thorburn on an earlier, similar proposal that was not supported by the telescope time assignment committees. They are grateful to W. J. Schuster for supplying new Strömgren photometry ahead of publication, and to A. Pedrosa for obtaining the WHT service observation. They also record their thanks to the Director and staff of the Anglo-Australian Observatory and the Australian Time Assignment Committee for the provision of facilities during this investigation. S. G. R. expresses his thanks to the Institute of Astronomy, Cambridge, for the use of facilities following the closure of the Royal Greenwich Observatory. T. C. B. acknowledges partial support for this work from grants AST 90-1376, AST 92-22326, INT 94-17547, and AST 95-29454 awarded by the National Science Foundation.
Akritas, M. G., \& Bershady, M. A. 1996, ApJ, 470, 706

Alonso, A., Arribas, S., \& Martinez-Roger, C. 1996a, A\&A, 313, 873 1996b, A\&AS, 117, 227

Beers, T. C., Flynn, K., \& Gebhardt, K. 1990, AJ, 100, 32

Beers, T. C., Preston, G. W., \& Shectman, S. A. 1992, AJ, 103, 1987

Beers, T. C., Rossi, S., Norris, J. E., Ryan, S. G., Molaro, P., \& Rebolo, R. 1998, Space Sci. Rev., 84, 139

Beers, T. C., Rossi, S., Norris, J. E., Ryan, S. G., \& Shefler, T. 1999, AJ, 117, 981

Bell, R. A., \& Oke, J. B. 1986, ApJ, 307, 253

Bevington, P. R. 1969, Data Reduction and Error Analysis for the Physical Sciences (New York: McGraw-Hill)

Boesgaard, A. M. 1985, PASP, 97, 784

Boesgaard, A. M., Deliyannis, C. P., Stephens, A., \& King, J. R. 1998, ApJ, 493, 206

Bonifacio, P., \& Molaro, P. 1997, MNRAS, 285, 847

. 1998, ApJ, 500, L175

Briley, M. M., Smith, V. V., Suntzeff, N. B., Lambert, D. L., Bell, R. A., \& Hesser, J. E. 1996, Nature, 383, 604

Burbidge, G., \& Hoyle, F. 1998, ApJ, 509, L1

Burstein, D., \& Heiles, C. 1982, AJ, 87, 1165

Carlsson, M., Rutten, R. J., Bruls, J. H. M. J., \& Shchukina, N. G. 1994, A\&A, 288, 860

Carney, B. W., Latham, D. W., Laird, J. B., \& Aguilar, L. A. 1994, AJ, 107, 2240

Cayrel, R. 1988, in IAU Symp. 132, The Impact of Very High S/N Spectroscopy on Stellar Physics, ed. G. Cayrel de Strobel \& M. Spite (Dordrecht: Kluwer), 345

Da Costa, G. S., \& Demarque, P. 1982, ApJ, 259, 193

D'Antona, F., \& Matteucci, F. 1991, A\&A, 248, 62

Deliyannis, C. P. 1995, in The Light Element Abundances, ed. P. Crane (Berlin: Springer), 395

Deliyannis, C. P., Boesgaard, A. M., \& King, J. R. 1995, ApJ, 452, L13

Deliyannis, C. P., Demarque, P., \& Kawaler, S. D. 1990, ApJS, 73, 21

Deliyannis, C. P., \& Malaney, R. A. 1995, ApJ, 453, 810

Deliyannis, C. P., Pinsonneault, M. H., \& Duncan, D. K. 1993, ApJ, 414, 740

Eggen, O. J. 1980, ApJS, 43, 457 1987, AJ, 93, 379

Fields, B. D., \& Olive, K. A. 1999, NewA, in press

Gratton, R. G., Carretta, E., \& Castelli, F. 1996, A\&A, 314, 191

Hobbs, L. M., \& Duncan, D. K. 1987, ApJ, 317, 796

Hobbs, L. M., \& Pilachowski, C. 1988, ApJ, 326, L23

Hobbs, L. M., \& Thorburn, J. A. 1991, ApJ, 375, 116 1994, ApJ, 428, L25 1997, ApJ, 491, 772

Hobbs, L. M., Welty, D. E., \& Thorburn, J. A. 1991, ApJ, 373, L47

King, J. R., Deliyannis, C. P., \& Boesgaard, A. M. 1996, AJ, 112, 2839

King, J. R., Stephens, A., Boesgaard, A. M., \& Deliyannis, C. P. 1998, AJ, 115,666

Kiselman, D. 1997, ApJ, 489, L107

Kurucz, R. L. 1993, CD -ROM 13, ATLAS9 Stellar Atmosphere Programs and $2 \mathrm{~km} / \mathrm{s}$ Grid (Cambridge: Smithsonian Astrophys. Obs.)

Lambert, D. L. 1995, A\&A, 301, 478

Levine, D. M., Berenson, M. L., \& Stephan, D. 1998, Statistics for Managers (London: Prentice Hall)

\section{REFERENCES}

Li, G. 1985, in Exploring Data Tables, Trends, and Shapes, ed. D. C. Hoaglin, F. Mosteller, \& J. W. Tukey (New York: Wiley), 281

Lucke, P. B. 1978, A\&A, 64, 367

Magain, P. 1987, A\&A, 181, 323

Molaro, P., Primas, F., \& Bonifacio, P. 1995, A\&A, 295, L47

Norris, J. 1981, ApJ, 248, 177

Norris, J. E. 1999, in ASP Conf. Ser., The Galactic Halo, ed. B. K. Gibson,

T. S. Axelrod, \& M. E. Putman (San Francisco: ASP), in press

Norris, J. E., \& Da Costa, G. S. 1995, ApJ, 447, 680

Norris, J. E., Ryan, S. G., \& Beers, T. C. 1997, ApJ, 488, 350

Norris, J. E., Ryan, S. G., Beers, T. C., \& Deliyannis, C. P. 1997a, ApJ, 485, 370

Norris, J. E., Ryan, S. G., \& Stringfellow, G. S. 1994, ApJ, 423, 386

Olive, K. A., \& Schramm, D. N. 1992, Nature, 360, 439

Peterson, R. C. 1983, ApJ, 275, 737

Peterson, R. C., Tarbell, T. D., \& Carney, B. W. 1983, ApJ, 265, 972

Pilachowski, C. A., Hobbs, L. M., \& De Young, D. S. 1989, ApJ, 345, L39

Pinsonneault, M. H., Deliyannis, C. P., \& Demarque, P. 1992, ApJS, 78, 179

Pinsonneault, M. H., Walker, T. P., Steigman, G., \& Narayanan, V. K. 1998, preprint (astro-ph/9803073)

Prantzos, N., Casse, M., \& Vangioni-Flam, E. 1993, ApJ, 403, 630

Ramaty, R., Kozlovsky, B., \& Lingenfelter, R. E. 1996, ApJ, 456, 525

Ramaty, R., Kozlovsky, B., Lingenfelter, R. E., \& Reeves, H. 1997, ApJ, 488, 730

Rebolo, R., Molaro, P., \& Beckman, J. E. 1988, A\&A, 192, 192

Rousseeuw, P. J., \& Leroy, A. M. 1987, Robust Regression and Outlier Detection (New York: Wiley)

Ryan, S. G. 1989, AJ, 98, 1693

. 1995, in The Light Element Abundances, ed. P. Crane (Berlin: Springer), 276

. 1996, in ASP Conf. Ser. 92, Formation of the Galactic HaloInside and Out, ed. H. Morrison \& A. Sarajedini (San Francisco: ASP), 113

Ryan, S. G., Beers, T. C., Deliyannis, C. P., \& Thorburn, J. A. 1996a, ApJ, 458,543

Ryan, S. G., \& Norris, J. E. 1991, AJ, 101, 1835

Ryan, S. G., Norris, J. E., \& Beers, T. C. 1996b, ApJ, 471, 254 1998, ApJ, 506, 892

Ryan, S. G., Norris, J. E., \& Bessell, M. S. 1991, AJ, 102, 303

Sandage, A., \& Fouts, G. 1987, AJ, 93, 74

Sandage, A., \& Kowal, C. 1986, AJ, 91, 1140

Savage, B. D., \& Mathis, J. S. 1979, ARA\&A, 17, 73

Schuster, W. J. \& Nissen, P. E. 1988, A\&AS, 73, 225

1989, A\&A, 222, 65

Schuster, W. J., Nissen, P. E., Parrao, L., Beers, T. C., \& Overgaard, L. P. 1996, A\&AS, 117, 317

Schuster, W. J., Parrao, L., \& Contreras Martinez, M. E. 1993, A\&AS, 97, 951

Searle, L., \& Zinn, R. 1978, ApJ, 225, 357

Siegel, S., \& Castellan, N. J. 1988, Nonparametric Statistics for the Behavioral Sciences (2d ed.; New York: McGraw-Hill)

Smith, V. V., Lambert, D. L., \& Nissen, P. E. 1993, ApJ, 408, 262

. 1998, ApJ, 506, 405

Sneden, C., Kraft, R. P., Shetrone, M. D., Smith, G. H., Langer, G. E., \&

Prosser, C. F. 1997, AJ, 114, 1964 
Spite, F., \& Spite, M. 1982, A\&A, 115, 357

1986, A\&A, 163, 140

1993, A\&A, 279, L9

Spite, M., Francois, P., Nissen, P. E., \& Spite, F. 1996, A\&A, 307, 172

Spite, M., Maillard, J. P., \& Spite, F. 1984, A\&A, 141, 56

Spite, M., Spite, F., Peterson, R. C., \& Chaffee, F. H., Jr. 1987, A\&A, 172, L9

Steigman, G., \& Walker, T. P. 1992, ApJ, 385, L13
Suntzeff, N. B. 1981, ApJS, 47, 1

Suntzeff, N. B., \& Smith, V. V. 1991, ApJ, 381, 160

Thorburn, J. A. 1994, ApJ, 421, 318

Thorburn, J. A., \& Beers, T. C. 1993, ApJ, 404, L13

Uitenbroek, H. 1998, ApJ, 498, 427

Vauclair, S. 1999, preprint (astro-ph/9902144)

Vauclair, S., \& Charbonnel, C. 1995, A\&A, 295, 715

Yoshii, Y., Kajino, T., \& Ryan, S. G. 1997, ApJ, 485, 605 